\title{
In Vitro Assessment of \\ Fluoropyrimidine-Metabolizing Enzymes: Dihydropyrimidine Dehydrogenase, Dihydropyrimidinase, and $\beta$-Ureidopropionase
}

\author{
Eiji Hishinuma ${ }^{1,2,3}$, Evelyn Gutiérrez Rico ${ }^{1}$ (D) and Masahiro Hiratsuka 1,2,3,4,* \\ 1 Laboratory of Pharmacotherapy of Life-Style Related Diseases, Graduate School of Pharmaceutical Sciences, \\ Tohoku University, Sendai 980-8578, Japan; ehishi@ingem.oas.tohoku.ac.jp (E.H.); \\ gutierrez@tohoku.ac.jp (E.G.R.) \\ 2 Tohoku Medical Megabank Organization, Tohoku University, Sendai 980-8573, Japan \\ 3 Advanced Research Center for Innovations in Next-Generation Medicine, Tohoku University, \\ Sendai 980-8573, Japan \\ 4 Department of Pharmaceutical Sciences, Tohoku University Hospital, Sendai 980-8574, Japan \\ * Correspondence: masahiro.hiratsuka.a8@tohoku.ac.jp; Tel.: +81-22-717-7049
}

Received: 12 June 2020; Accepted: 21 July 2020; Published: 22 July 2020

\begin{abstract}
Fluoropyrimidine drugs (FPs), including 5-fluorouracil, tegafur, capecitabine, and doxifluridine, are among the most widely used anticancer agents in the treatment of solid tumors. However, severe toxicity occurs in approximately $30 \%$ of patients following FP administration, emphasizing the importance of predicting the risk of acute toxicity before treatment. Three metabolic enzymes, dihydropyrimidine dehydrogenase (DPD), dihydropyrimidinase (DHP), and $\beta$-ureidopropionase ( $\beta$-UP), degrade FPs; hence, deficiencies in these enzymes, arising from genetic polymorphisms, are involved in severe FP-related toxicity, although the effect of these polymorphisms on in vivo enzymatic activity has not been clarified. Furthermore, the clinical usefulness of current methods for predicting in vivo activity, such as pyrimidine concentrations in blood or urine, is unknown. In vitro tests have been established as advantageous for predicting the in vivo activity of enzyme variants. This is due to several studies that evaluated FP activities after enzyme metabolism using transient expression systems in Escherichia coli or mammalian cells; however, there are no comparative reports of these results. Thus, in this review, we summarized the results of in vitro analyses involving DPD, DHP, and $\beta$-UP in an attempt to encourage further comparative studies using these drug types and to aid in the elucidation of their underlying mechanisms.
\end{abstract}

Keywords: fluoropyrimidine; dihydropyrimidine dehydrogenase; dihydropyrimidinase; $\beta$-ureidopropionase; genetic polymorphism

\section{Introduction}

Fluoropyrimidine drugs (FPs), including 5-fluorouracil (5-FU) and its oral prodrugs tegafur, capecitabine, and doxifluridine, are widely used in the treatment of solid tumors in the gastrointestinal tract, breast, liver, lung, head, and neck [1-3]. FP-based treatments have a narrow therapeutic index, which has led to severe adverse effects in approximately $30 \%$ of cancer patients, including mucositis, diarrhea, neutropenia, thrombocytopenia, and hand-foot syndrome [4-8]. Additionally, severe treatment toxicities could lead to treatment interruption, which increases the subsequent risk of therapeutic failure as well as patient death [9].

Genetic polymorphisms of thymidylate synthase (TYMS), methylene tetrahydrofolate reductase (MTHFR), and miR-27a are associated with the development of severe toxicities as well as treatment 
resistance; however, FP-related toxicity is mainly dependent on FP catabolism. Over $80 \%$ of an administered dose of 5-FU is rapidly degraded by three consecutive enzymes belonging to the endogenous pyrimidine, uracil, and thymine catabolic pathways (Figure 1), the only known 5-FU in vivo degradation pathway. Initially, the rate-limiting enzyme dihydropyrimidine dehydrogenase (DPD, EC 1.3.1.2), mainly found in the liver, catalyzes the reduction of 5-FU to dihydro-5-fluorouracil $\left(\mathrm{FUH}_{2}\right)$. Subsequently, dihydropyrimidinase (DHP, EC 3.5.2.2) catalyzes the hydrolytic ring opening of $\mathrm{FUH}_{2}$ to form fluoro- $\beta$-ureidopropionic acid (FUPA). Even though DPD and DHP catalysis is reversible, the positive reaction is dominant in vivo [10-15]. Lastly, $\beta$-ureidopropionase ( $\beta$-UP, EC 3.5.1.6) catalyzes the hydrolysis of FUPA to fluoro- $\beta$-alanine. The three enzymes (DPD, DHP, and $\beta$-UP) are encoded by the DPYD, DPYS, and UPB1 genes, respectively [16-18].<smiles>NCCC(=O)O</smiles><smiles>NCC(F)C(=O)O</smiles>

Figure 1. Uracil and 5-fluorouracil degradation pathway. Uracil and 5-fluorouracil are catabolized successively by dihydropyrimidine dehydrogenase, dihydropyrimidinase, and $\beta$-ureidopropionase. $\beta$-Alanine and fluoro- $\beta$-alanine are the final metabolites in this pathway.

Decreased DPD and DHP enzymatic activities have been linked to genetic polymorphisms identified in patients with severe FP-related toxicities; for each causative polymorphism, the reduction in activity is caused mainly by the substitution or deletion of amino acids [19-21]. However, the relationship between $\beta$-UP activity and the development of FP-related toxicity is still unknown. To date, the specific effects of previously identified polymorphisms on enzymatic function are largely unknown. Only four DPYD variants (c.1905 + 1G > A (IVS14 + 1G > A, DPYD*2A); c.1679T > G $\left(D_{P Y D}^{*} 13\right.$, p.I560S); c.1129 - 5923C > G /hapB3; and c.2846A > T (p.D949V)) have been characterized as predictive markers for FP-related toxicity in Caucasians [22]. However, significant racial and individual differences in polymorphism location and frequency make it challenging to safely extrapolate the clinical data and institute regional guidelines from one population to another. Thus, it is necessary to further clarify the effects of genetic polymorphisms in an attempt to establish their effect on in vivo enzymatic function. For example, before FP administration, PCR-Restriction Fragment Length Polymorphism (RFLP) analysis, Sanger sequencing, and next-generation sequencing analysis are often used for detecting genetic polymorphisms and establishing patient risk. Moreover, hepatic DPD activity, and thus DPD deficiency incidence, can be predicted by assessing peripheral blood mononuclear cell (PBMC) DPD activity. However, to date, there are no established methods to quantify DHP and $\beta$-UP activity clinically.

The most direct method to understand the effect of the genetic polymorphisms of these enzymes on FP pharmacokinetics is to measure metabolite concentrations in blood and urine from subjects with the respective genotypes after FP administration. However, in vivo testing is highly invasive due to 
continuous blood sampling and poses a considerable risk of FP-related toxicity. Additionally, as the variants of interest are mainly low-frequency polymorphisms, the recruitment of an adequate subject pool to obtain statistically significant data is considerably difficult. While pyrimidine metabolites in blood and urine have been previously quantified to assess enzymatic activity in vivo, these have yielded contradictory results [23,24].

In contrast, in vitro testing using heterologous expression systems has yielded reproducible results using non-invasive methods to facilitate enzymatic activity assessment [25]. Amongst these, several in vitro FP analyses using Escherichia coli or mammalian cells have been reported. While other in vitro techniques have been used to evaluate genetic polymorphisms including gene expression profiling, in this review, we focus on the in vitro analysis of the FP-metabolizing enzymes: DPD, DHP, and $\beta$-UP, thus providing further information to aid in the application of genetic testing in a clinical setting in light of recent novel insights.

\section{Dihydropyrimidine Dehydrogenase (DPD)}

$\mathrm{DPD}$, the rate-limiting enzyme of the pyrimidine degradation pathway, catalyzes the reduction of 5-FU and uracil to $\mathrm{FUH}_{2}$ and dihydrouracil $\left(\mathrm{UH}_{2}\right)$. The DPD gene (DPYD) is expressed in most human tissues, but the expression level is highest in the liver and PBMCs [26]. Located on chromosome $1 \mathrm{p} 21$, human DPYD is comprised of 23 exons and features a $3078 \mathrm{bp}$ open reading frame, encoding a polypeptide containing 1025 amino acid residues [27].

DPD deficiency is an autosomal recessive disorder first reported in a child with neurological symptoms by Bakkeren et al., which was characterized by the accumulation of uracil and thymine in urine, blood, and cerebrospinal fluid [28]. The clinical symptoms include convulsions, autism, microcephaly, growth impairment, and intellectual disability, although asymptomatic cases have also been reported [29-31]. The frequency of DPD-deficient patients varies greatly across world populations. While Caucasian frequencies range from 3-5\% for partial deficiency and $0.2 \%$ for complete deficiency, it is estimated to be extremely rare in Asians [32,33]. In the case of asymptomatic DPD deficiency, there is a considerable risk of FP accumulation during treatment, including 5-FU, which could lead to severe toxicity in patients [34-36]. Therefore, it is imperative to diagnose DPD deficiency before chemotherapy administration, even in cases with no prior clinical evidence of this condition.

Of the three metabolic enzymes, DPYD is the most studied gene. More than 500 DPYD polymorphisms to date have been identified and have been linked to FP-related toxicity in cancer patients [22,37-45]. Several of these variants are known to alter amino acid sequence or mRNA splicing, resulting in decreased enzymatic activity. Within the Clinical Pharmacogenetics Implementation Consortium (CPIC) guidelines, three variants (c.85T > C (DPYD*9A, p.C29R), c.1627A > G (DPYD*5, p.I543V), and c.2194G > A (DPYD*6, p.V732I)) are reported to have no effect on enzyme activity [46]. Four variants that cause exon 14 skipping or amino acid substitution (c.1905 + 1G > A (IVS14 + 1G > A, DPYD*2A), c.1679T > G (DPYD*13, p.I560S), c.1129 - 5923C > G/hapB3, and c.2846A > T (p.D949V)) are designated as having reduced enzymatic function and thus increase the risk of developing toxicity. Similarly, the Dutch Pharmacogenetics Working Group (DPWG) guidelines define these same four variants as risk factors for FP-related toxicity and recommend reducing treatment dosage when a patient possesses one of them [47]. Although DPYD*9A, ${ }^{*}$, and ${ }^{*} 6$ are common variants in many ethnic groups, these four risk variants have not yet been identified in Asians [48-50].

For most identified DPYD variants, except those mentioned above, the effect on DPD activity is unknown, and it is important to clarify the DPD phenotype [51]. The current standard to predict DPD activity measures its enzymatic activity in PBMCs, which correlates with hepatic DPD activity [52,53]. However, this method is not easily implemented in its current form in routine medical care, as it lacks solid evidence of clinical utility. Due to insufficient sensitivity, methods for quantifying pyrimidine metabolites in blood or urine might not identify patients with partial DPD deficiencies [23]. Moreover, additional studies on the clinical validity and utility of these tests are required before implementation can be justified. 
In vitro testing is one of the methods used for estimating DPD phenotypes and for the functional analysis of identified non-synonymous variants [54-60]. Several studies of such tests using E. coli or mammalian cell expression systems have been reported (Table 1). Ogura et al. functionally analyzed two variants (G366A and T768K) identified from 150 healthy Japanese volunteers using an E. coli expression system [57]. Interestingly, while the G366A mutation produced a decreased intrinsic clearance $\left(C L_{i n t}\right)$ for 5 -FU, reducing DPD activity by $50 \%$, the T768K mutation did not. However, T768K-related activity decreased at a faster rate than that of wild-type DPD, suggesting protein instability. In a subsequent study, Offer et al. expressed 80 non-synonymous variants in HEK293T/c17 cells and measured their enzymatic activities using 5-FU as a substrate [58]. M166V, E828K, K861R, and P1023T exhibited significantly higher activity than wild-type DPD. In contrast, 31 variants, including D949V, exhibited significantly lower activity than wild-type DPD. Elraiyah et al. also analyzed 10 non-synonymous variants identified from 588 Somali and Kenyan individuals using HEK293T/c17 cells [59], in which P86L, P237L, A513V, T793I, V941A, and P1023S exhibited significantly reduced DPD activities. We have characterized 21 DPD allelic variants identified from 1070 Japanese individuals by transient expression in 293FT cells [60]. Among these, 10 (T298M, V313L, V335M, A380V, V434L, V515I, R592W, T768K, H807R, and V826M) showed significantly reduced $C L_{\text {int }}$ values relative to wild-type DPD, and the 5-FU metabolic activity of G926V was practically zero. These reports have yielded consistent results for $D P Y D^{*} 2 A$, which exhibited decreased activity, and for DPYD*5 (I543V) and ${ }^{*} 6$ (V732I), which exhibited activities that were not considerably different from that of wild-type DPD. In contrast, there are variants such as $D P Y D^{*} 9 A(C 29 \mathrm{R})$ and M166V, whose reported activities differ significantly among previous reports. Ogura et al. and our group found that M166V had a lower activity compared with that of wild-type DPD, while Offer et al. reported a reduction in activity for M166V. The differences in these activities are believed to be due to the differences in assay conditions and cell lines used. Notably, we and Ogura et al. reported DPD variants that were identified almost exclusively in Japanese individuals. Therefore, this raises awareness of the possibility of unidentified rare and relevant ethno-specific variants, which could lead to severe FP-related toxicity.

From a biochemical perspective, human DPD is a flavoprotein containing a single flavin mononucleotide (FMN), a single flavin adenine dinucleotide (FAD), and four iron-sulfur (FeS) clusters. Human DPD consists of five major domains [61-64]. Domain I (residues 27-172) and domain V (residues 1-26, 848-1025) each contain two FeS clusters. FAD- and nicotinamide adenine dinucleotide phosphate (NADPH)-binding sites are located in domain II (residues 173-286, 442-524) and domain III (residues 287-441), respectively. FMN and the substrate both bind to domain IV (residues 525-847). Human DPD form a dimer, in which electrons from NADPH are transferred to the FeS clusters to catalyze the reduction of bound substrates [65]. Domains II and IV are essential for DPD activity in the structural analysis of variants. Amino acid substitutions that have been observed to affect protein conformation adjacent to the FeS clusters have also caused a significant decrease in enzyme activity.

Henricks et al. described a prediction method using an activity score system and divided DPYD alleles into three categories, consisting of fully functional alleles (wild-type; value of 1), reduced activity alleles (c.2846A > T and HapB3; value of 0.5$)$, and nonfunctional alleles (DPYD*2A and ${ }^{*} 13$; value of 0) [66]. Allele values are totaled for a given patient, leading to an individual gene activity score that represents the DPD phenotype of the patient. Moreover, Shrestha et al. developed a $D P Y D$-specific variant classifier (DPYD-Varifier) using machine learning of in vitro functional data from 156 variants [67]. This model exhibited an accuracy of $85 \%$ and outperformed other in silico prediction tools, including PROVEAN, SIFT, and Polyphen-2. In the future, it may be possible to easily predict in vivo DPD activity using machine learning by creating compound databases by gathering detailed information from in vitro analyses. Recently, a list of DPYD variants has been added to the Pharmacogene Variation Consortium website (https://www.pharmvar.org/gene/DPYD). It is expected that evidence-based decisions on FP therapeutic regimens and patient-specific dose guidelines could be applied on the basis of an activity score formula, as has been recommended and implemented with other clinically relevant metabolic enzymes. 
Table 1. DPYD variants reported in in vitro analysis.

\begin{tabular}{|c|c|c|c|c|c|c|c|c|c|}
\hline dbSNP rsID & PharmVar ID & Location & $\begin{array}{l}\text { Nucleotide } \\
\text { Change }\end{array}$ & $\begin{array}{l}\text { Amino Acid } \\
\text { Substitution }\end{array}$ & Domain & $\begin{array}{l}\text { Expression } \\
\text { System }\end{array}$ & Substrates & Effect & References \\
\hline rs150036960 & PV00901 & Exon 2 & $46 C>G$ & L16V & $\mathrm{V}$ & HEK293T/c17 & $5-\mathrm{FU}$ & Normal function & [58] \\
\hline rs72549310 & PV01042 & Exon 2 & $61 \mathrm{C}>\mathrm{T}$ & R21X & I & HEK293T/c17 & 5-FU & No function & [58] \\
\hline rs80081766 & PV01307 & Exon 2 & $62 \mathrm{G}>\mathrm{A}$ & $\mathrm{R} 21 \mathrm{Q}$ & I & HEK293T/c17 & 5-FU & Normal function & [58] \\
\hline- & - & Exon 2 & $74 \mathrm{~A}>\mathrm{G}$ & $\mathrm{H} 25 \mathrm{R}$ & I & 293FT & $5-\mathrm{FU}$ & $156 \%$ of $C L_{i n t}$ ratio & [60] \\
\hline \multirow{2}{*}{ rs1801265 } & \multirow{2}{*}{ PV00910 } & \multirow{2}{*}{ Exon 2} & $85 \mathrm{~T}>\mathrm{C}$ & \multirow{2}{*}{ C29R } & \multirow{2}{*}{ I } & HEK293T/c17 & $5-\mathrm{FU}$ & Increased function & [54] \\
\hline & & & $\left(D P Y D^{* 9 A}\right)$ & & & HEK293 Flp-In & Thymine & Decreased function & [55] \\
\hline rs371587702 & PV00962 & Exon 3 & $194 \mathrm{C}>\mathrm{T}$ & $\mathrm{T} 65 \mathrm{M}$ & I & HEK293T/c17 & 5-FU & Normal function & [58] \\
\hline- & - & Exon 4 & $257 \mathrm{C}>\mathrm{T}$ & P86L & I & HEK293T/c17 & 5-FU & No function & [59] \\
\hline rs72549309 & PV01041 & Exon 4 & $\begin{array}{c}\text { 295delTCAT } \\
\left(D P Y D^{*} 7\right)\end{array}$ & F100fs & I & HEK293T/c17 & $5-\mathrm{FU}$ & No function & [58] \\
\hline rs150385342 & PV00902 & Exon 4 & $313 G>A$ & A105T & I & HEK293T/c17 & 5-FU & Normal function & [58] \\
\hline- & - & Exon 5 & $325 \mathrm{~T}>\mathrm{A}$ & Y109N & I & $293 \mathrm{FT}$ & 5-FU & $79 \%$ of $C L_{\text {int }}$ ratio & [60] \\
\hline rs141462178 & PV00878 & Exon 5 & $343 \mathrm{~A}>\mathrm{G}$ & M115V & I & HEK293T/c17 & $5-\mathrm{FU}$ & Normal function & [58] \\
\hline \multirow{3}{*}{ rs200562975 } & \multirow{3}{*}{ PV00927 } & \multirow{3}{*}{ Exon 5} & \multirow{3}{*}{$451 \mathrm{~A}>\mathrm{G}$} & \multirow{3}{*}{ N151D } & \multirow{3}{*}{ I } & 293FT & 5-FU & $107 \%$ of $C L_{\text {int }}$ ratio & [60] \\
\hline & & & & & & HEK293T/c17 & 5-FU & Normal function & [58] \\
\hline & & & & & & 293FT & 5-FU & $77 \%$ of $C L_{i n t}$ ratio & [60] \\
\hline rs2297595 & PV0943 & Exon 6 & $496 \mathrm{~A}>\mathrm{G}$ & M166V & I & HEK293T/c17 & 5-FU & Increased function & [58] \\
\hline rs371792178 & - & Exon 6 & $524 \mathrm{C}>\mathrm{T}$ & S175L & II & 293FT & 5-FU & $131 \%$ of $C L_{i n t}$ ratio & [60] \\
\hline rs115232898 & PV00862 & Exon 6 & $557 \mathrm{~A}>\mathrm{G}$ & Y186C & II & HEK293T/c17 & 5-FU & Decreased function & [58] \\
\hline rs72549308 & PV01040 & Exon 6 & $601 \mathrm{~A}>\mathrm{C}$ & S201R & II & HEK293T/c17 & $5-\mathrm{FU}$ & No function & [58] \\
\hline rs72549307 & PV01039 & Exon 6 & $632 \mathrm{~A}>\mathrm{G}$ & Y211C & II & HEK293T/c17 & $5-\mathrm{FU}$ & Decreased function & [58] \\
\hline rs1801266 & PV00911 & Exon 7 & $\begin{array}{c}703 C>\mathrm{T} \\
\left(D P Y D^{*} 8\right)\end{array}$ & R235W & II & HEK293T/c17 & 5-FU & Decreased function & [58] \\
\hline rs780025995 & PV01299 & Exon 7 & $710 \mathrm{C}>\mathrm{T}$ & P237L & II & HEK293T/c17 & 5-FU & Decreased function & [59] \\
\hline rs45589337 & PV00984 & Exon 8 & $775 \mathrm{~A}>\mathrm{G}$ & K259E & II & HEK293T/c17 & 5-FU & Normal function & [58] \\
\hline rs777220476 & PV01275 & Exon 9 & $851 \mathrm{G}>\mathrm{T}$ & $\mathrm{G} 284 \mathrm{~V}$ & II & HEK293 Flp-In & Thymine & No function & [56] \\
\hline rs146356975 & PV00895 & Exon 9 & $868 \mathrm{~A}>\mathrm{G}$ & K290E & III & HEK293T/c17 & $5-F U$ & Decreased function & [58] \\
\hline rs143878757 & PV00886 & Exon 9 & $893 \mathrm{C}>\mathrm{T}$ & $\mathrm{T} 298 \mathrm{M}$ & III & $293 \mathrm{FT}$ & $5-\mathrm{FU}$ & $50 \%$ of $C L_{\text {int }}$ ratio & [60] \\
\hline rs183105782 & PV00914 & Exon 9 & $910 \mathrm{~T}>\mathrm{C}$ & $\mathrm{Y} 304 \mathrm{H}$ & III & HEK293T/c17 & 5-FU & Decreased function & [58] \\
\hline rs150437414 & PV00904 & Exon 9 & $929 \mathrm{~T}>\mathrm{C}$ & L310S & III & HEK293T/c17 & 5-FU & Normal function & [58] \\
\hline rs145112791 & PV00891 & Exon 9 & $934 \mathrm{C}>\mathrm{T}$ & L312F & III & HEK293T/c17 & 5-FU & Normal function & [58] \\
\hline- & - & Exon 9 & $937 \mathrm{G}>\mathrm{T}$ & V313L & III & 293FT & $5-\mathrm{FU}$ & $30 \%$ of $C L_{\text {int }}$ ratio & [60] \\
\hline rs201018345 & PV00933 & Exon 10 & $967 \mathrm{G}>\mathrm{A}$ & A323T & III & HEK293T/c17 & $5-\mathrm{FU}$ & Normal function & [58] \\
\hline
\end{tabular}


Table 1. Cont.

\begin{tabular}{|c|c|c|c|c|c|c|c|c|c|}
\hline dbSNP rsID & PharmVar ID & Location & $\begin{array}{l}\text { Nucleotide } \\
\text { Change }\end{array}$ & $\begin{array}{l}\text { Amino Acid } \\
\text { Substitution }\end{array}$ & Domain & $\begin{array}{l}\text { Expression } \\
\text { System }\end{array}$ & Substrates & Effect & References \\
\hline rs72549306 & PV01038 & Exon 10 & $1003 \mathrm{G}>\mathrm{A}$ & V335M & III & 293FT & 5-FU & $47 \%$ of $C L_{i n t}$ ratio & [60] \\
\hline rs72549306 & PV01037 & Exon 10 & $\begin{array}{l}1003 \mathrm{G}>\mathrm{T} \\
\left(D P Y D^{*} 11\right)\end{array}$ & V335L & III & HEK293T/c17 & $5-\mathrm{FU}$ & Normal function & [58] \\
\hline rs183385770 & PV00915 & Exon 10 & $1024 \mathrm{G}>\mathrm{A}$ & D342N & III & HEK293T/c17 & 5-FU & Decreased function & [58] \\
\hline rs190577302 & PV00919 & Exon 10 & $1054 C>G$ & L352V & III & HEK293T/c17 & $5-\mathrm{FU}$ & Decreased function & [58] \\
\hline rs143154602 & PV00882 & Exon 10 & $1057 \mathrm{C}>\mathrm{T}$ & R353C & III & HEK293T/c17 & 5-FU & No function & [58] \\
\hline- & - & Exon 10 & $1097 \mathrm{G}>\mathrm{C}$ & G366A & III & $\begin{array}{c}\text { 293FT } \\
\text { Escherichia coli }\end{array}$ & $\begin{array}{l}5-\mathrm{FU} \\
5-\mathrm{FU}\end{array}$ & $\begin{array}{l}71 \% \text { of } C L_{i n t} \text { ratio } \\
47 \% \text { of } C L_{\text {int }} \text { ratio }\end{array}$ & {$[60]$} \\
\hline- & - & Exon 11 & $1139 \mathrm{C}>\mathrm{T}$ & A380V & III & 293FT & 5-FU & $33 \%$ of $C L_{\text {int }}$ ratio & [60] \\
\hline- & - & Exon 11 & $1150 \mathrm{~A}>\mathrm{G}$ & K384E & III & $293 \mathrm{FT}$ & 5-FU & $68 \%$ of $C L_{i n t}$ ratio & [60] \\
\hline rs78060119 & PV01302 & Exon 11 & $\begin{array}{c}1156 \mathrm{G}> \\
\mathrm{T}\left(D^{\prime} Y D^{*} 12\right)\end{array}$ & E386X & III & HEK293T/c17 & $5-\mathrm{FU}$ & No function & [58] \\
\hline rs140602333 & PV00874 & Exon 11 & $1180 \mathrm{C}>\mathrm{T}$ & R394W & III & HEK293T/c17 & 5-FU & Normal function & [58] \\
\hline rs143815742 & PV00883 & Exon 11 & $1181 \mathrm{G}>\mathrm{T}$ & R394L & III & HEK293T/c17 & 5-FU & Normal function & [58] \\
\hline rs143815742 & PV00884 & Exon 11 & $1181 G>A$ & R394Q & III & HEK293T/c17 & 5-FU & Normal function & [59] \\
\hline- & - & Exon 11 & $1201 G>A$ & G401R & III & HEK293 Flp-In & Thymine & Decreased function & [55] \\
\hline rs61622928 & PV01018 & Exon 11 & $1218 \mathrm{G}>\mathrm{A}$ & M406I & III & $\begin{array}{l}\text { HEK293T/c17 } \\
\text { HEK293 Flp-In }\end{array}$ & $\begin{array}{l}\text { 5-FU } \\
\text { Thymine }\end{array}$ & $\begin{array}{l}\text { Normal function } \\
\text { Normal function }\end{array}$ & {$[58]$} \\
\hline rs764666241 & PV0183 & Exon 11 & $1278 \mathrm{G}>\mathrm{T}$ & M426I & III & HEK293T/c17 & 5-FU & Normal function & [58] \\
\hline rs200693895 & PV00931 & Exon 11 & $1280 \mathrm{~T}>\mathrm{C}$ & V427A & III & HEK293 Flp-In & Thymine & Normal function & [56] \\
\hline rs142512579 & PV00880 & Exon 11 & $1294 \mathrm{G}>\mathrm{A}$ & $\mathrm{D} 432 \mathrm{~N}$ & III & HEK293T/c17 & $5-F U$ & Normal function & [58] \\
\hline- & - & Exon 11 & $1300 \mathrm{G}>\mathrm{C}$ & V434L & III & $293 \mathrm{FT}$ & 5-FU & $44 \%$ of $C L_{\text {int }}$ ratio & [60] \\
\hline rs186169810 & PV00916 & Exon 11 & $1314 \mathrm{~T}>\mathrm{G}$ & F438L & III & HEK293T/c17 & $5-\mathrm{FU}$ & Decreased function & [58] \\
\hline rs72975710 & PV01043 & Exon 12 & $1349 \mathrm{C}>\mathrm{T}$ & $\mathrm{A} 450 \mathrm{~V}$ & II & HEK293T/c17 & $5-\mathrm{FU}$ & Normal function & [58] \\
\hline rs144395748 & PV00888 & Exon 12 & $1358 \mathrm{C}>\mathrm{G}$ & P453R & II & HEK293T/c17 & 5-FU & Normal function & [58] \\
\hline rs199549923 & PV00921 & Exon 12 & $1403 C>A$ & $\mathrm{~T} 468 \mathrm{~N}$ & II & HEK293T/c17 & 5-FU & Normal function & [58] \\
\hline rs72549304 & PV01035 & Exon 12 & $1475 \mathrm{C}>\mathrm{T}$ & S492L & II & HEK293T/c17 & 5-FU & Decreased function & [58] \\
\hline rs111858276 & PV00857 & Exon 12 & $1484 \mathrm{~A}>\mathrm{G}$ & D495G & II & HEK293T/c17 & $5-\mathrm{FU}$ & Decreased function & [58] \\
\hline rs138391898 & PV00867 & Exon 12 & $1519 \mathrm{G}>\mathrm{A}$ & V507I & II & HEK293T/c17 & 5-FU & Normal function & [58] \\
\hline rs760663364 & PV01150 & Exon13 & $1538 \mathrm{C}>\mathrm{T}$ & A513V & II & HEK293T/c17 & 5-FU & Decreased function & [59] \\
\hline rs148994843 & PV00900 & Exon 13 & $1543 G>A$ & V515I & II & $\begin{array}{c}\text { 293FT } \\
\text { HEK293T/c17 }\end{array}$ & $\begin{array}{l}5-\mathrm{FU} \\
5-\mathrm{FU}\end{array}$ & $\begin{array}{l}36 \% \text { of } C L_{\text {int }} \text { ratio } \\
\text { Normal function }\end{array}$ & $\begin{array}{l}{[60]} \\
{[58]}\end{array}$ \\
\hline - & - & Exon 13 & $1567 \mathrm{C}>\mathrm{T}$ & L523F & II & HEK293T/c17 & 5-FU & Normal function & [59] \\
\hline
\end{tabular}


Table 1. Cont.

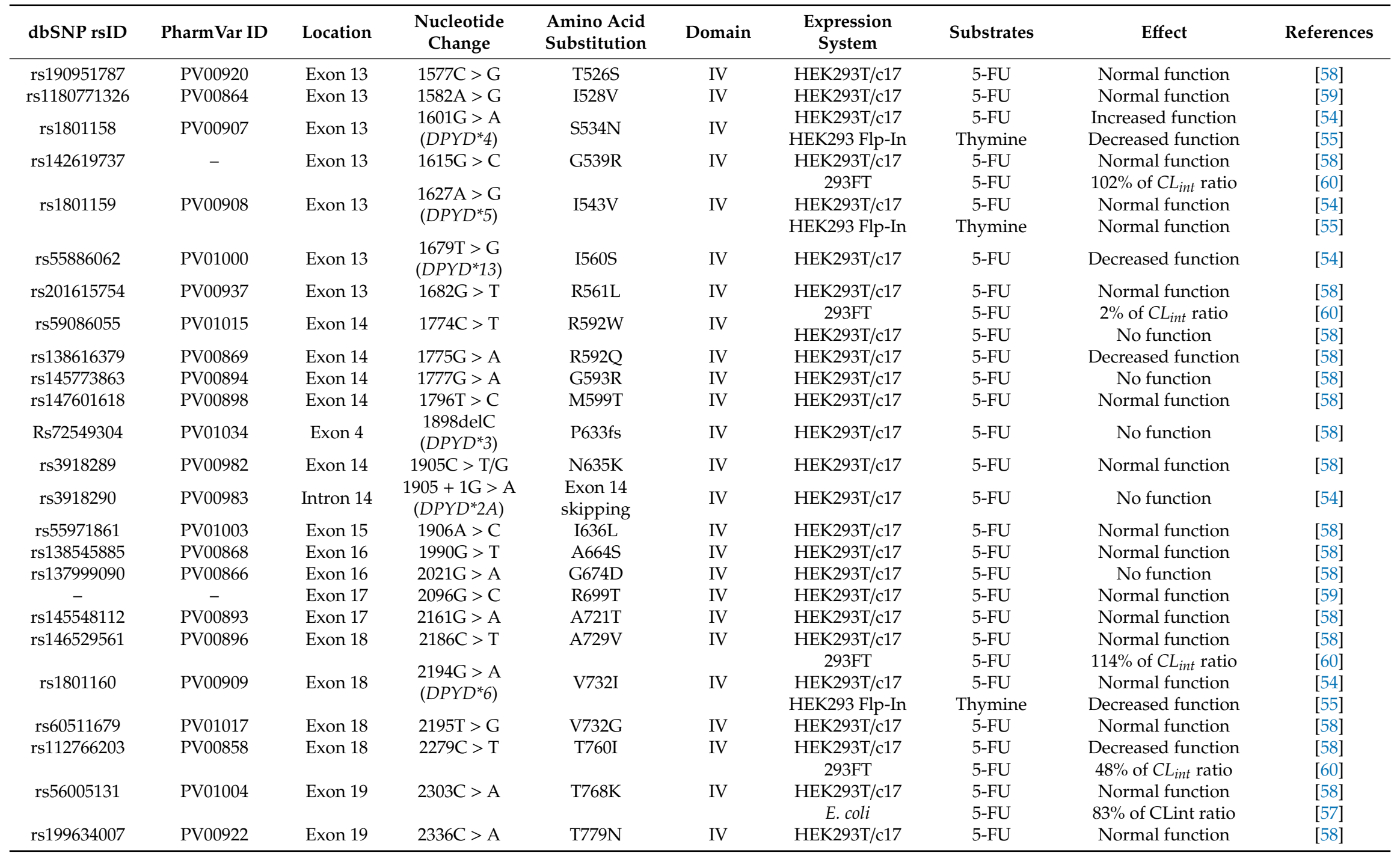


Table 1. Cont.

\begin{tabular}{|c|c|c|c|c|c|c|c|c|c|}
\hline dbSNP rsID & Pharm Var ID & Location & $\begin{array}{c}\text { Nucleotide } \\
\text { Change }\end{array}$ & $\begin{array}{l}\text { Amino Acid } \\
\text { Substitution }\end{array}$ & Domain & $\begin{array}{l}\text { Expression } \\
\text { System }\end{array}$ & Substrates & Effect & References \\
\hline rs547099198 & PV00994 & Exon 19 & $2378 \mathrm{C}>\mathrm{T}$ & T793I & IV & HEK293T/c17 & 5-FU & Decreased function & [59] \\
\hline- & - & Exon 19 & $2420 A>G$ & H807R & IV & $293 \mathrm{FT}$ & 5-FU & $50 \%$ of $C L_{\text {int }}$ ratio & [60] \\
\hline - & - & Exon 20 & $2476 \mathrm{G}>\mathrm{A}$ & V826M & IV & 293FT & 5-FU & $35 \%$ of $C L_{i n t}$ ratio & [60] \\
\hline rs200687447 & PV00930 & Exon 20 & $2482 \mathrm{G}>\mathrm{A}$ & E828K & IV & HEK293T/c17 & 5-FU & Increased function & [58] \\
\hline rs60139309 & PV01016 & Exon 20 & $2582 A>G$ & K861R & $\mathrm{V}$ & HEK293T/c17 & $5-\mathrm{FU}$ & Increased function & [58] \\
\hline rs201035051 & PV00934 & Exon 21 & $2623 \mathrm{~A}>\mathrm{C}$ & K875Q & $\mathrm{V}$ & HEK293T/c17 & 5-FU & Normal function & [58] \\
\hline rs55674432 & PV00996 & Exon 21 & $2639 \mathrm{G}>\mathrm{T}$ & G880V & $\mathrm{V}$ & HEK293T/c17 & $5-\mathrm{FU}$ & No function & [58] \\
\hline rs147545709 & PV00897 & Exon 21 & $2656 \mathrm{C}>\mathrm{T}$ & $\mathrm{R} 886 \mathrm{C}$ & $\mathrm{V}$ & HEK293T/c17 & $5-\mathrm{FU}$ & Normal function & [58] \\
\hline rs1801267 & PV00912 & Exon 21 & $\begin{array}{l}2657 \mathrm{G}>\mathrm{A} \\
\left(D P Y D^{*} 9 B\right)\end{array}$ & $\mathrm{R} 886 \mathrm{H}$ & V & HEK293T/c17 & $5-\mathrm{FU}$ & Normal function & [58] \\
\hline rs188052243 & PV00918 & Exon 21 & $2678 \mathrm{~A}>\mathrm{G}$ & N893S & V & $\begin{array}{c}\text { 293FT } \\
\text { HEK293T/c17 }\end{array}$ & $\begin{array}{l}5-\mathrm{FU} \\
5-\mathrm{FU}\end{array}$ & $\begin{array}{l}61 \% \text { of } C L_{\text {int }} \text { ratio } \\
\text { Decreased function }\end{array}$ & $\begin{array}{l}{[60]} \\
{[58]}\end{array}$ \\
\hline- & - & Exon 22 & $2777 \mathrm{G}>\mathrm{T}$ & G926V & $\mathrm{V}$ & 293FT & $5-\mathrm{FU}$ & No function & [58] \\
\hline - & - & Exon 22 & $2822 \mathrm{~T}>\mathrm{C}$ & V941A & $\mathrm{V}$ & HEK293T/c17 & $5-\mathrm{FU}$ & Decreased function & [59] \\
\hline- & - & Exon 22 & $2843 \mathrm{~T}>\mathrm{C}$ & $\mathrm{I} 948 \mathrm{~T}$ & $\mathrm{~V}$ & HEK293 Flp-In & Thymine & Decreased function & [56] \\
\hline rs67376798 & PV01031 & Exon 22 & $2846 \mathrm{~A}>\mathrm{T}$ & D949V & V & $\begin{array}{l}\text { HEK293T/c17 } \\
\text { HEK293 Flp-In }\end{array}$ & $\begin{array}{l}\text { 5-FU } \\
\text { Thymine }\end{array}$ & $\begin{array}{l}\text { Decreased function } \\
\text { Decreased function }\end{array}$ & $\begin{array}{l}{[58]} \\
{[55]}\end{array}$ \\
\hline rs141044036 & PV00876 & Exon 22 & $2872 A>G$ & K958E & $\mathrm{V}$ & HEK293T/c17 & $5-\mathrm{FU}$ & No function & [58] \\
\hline rs72547601 & PV01032 & Exon 23 & $2933 A>G$ & H978R & $\mathrm{V}$ & HEK293T/c17 & $5-\mathrm{FU}$ & No function & [58] \\
\hline rs61757362 & PV01019 & Exon 23 & $2948 \mathrm{C}>\mathrm{T}$ & T983I & $\mathrm{V}$ & HEK293T/c17 & $5-\mathrm{FU}$ & Decreased function & [58] \\
\hline rs202144771 & PV00941 & Exon 23 & $2977 \mathrm{C}>\mathrm{T}$ & L993F & $\mathrm{V}$ & HEK293T/c17 & $5-\mathrm{FU}$ & Normal function & [58] \\
\hline rs139459586 & PV00870 & Exon 23 & $2978 \mathrm{~T}>\mathrm{G}$ & L993R & V & HEK293T/c17 & $5-\mathrm{FU}$ & Normal function & [58] \\
\hline rs1801268 & PV00913 & Exon 23 & $\begin{array}{l}2983 \mathrm{G}>\mathrm{T} \\
\left(D P Y D^{* 10}\right)\end{array}$ & V995F & V & HEK293T/c17 & $5-\mathrm{FU}$ & No function & [58] \\
\hline rs140114515 & PV00873 & Exon 23 & $3049 \mathrm{G}>\mathrm{A}$ & V1017I & $\mathrm{V}$ & HEK293T/c17 & $5-\mathrm{FU}$ & Normal function & [58] \\
\hline rs148799944 & PV00899 & Exon 23 & $3061 G>C$ & V1021L & V & HEK293T/c17 & $5-\mathrm{FU}$ & Normal function & [58] \\
\hline rs114096998 & PV00860 & Exon 23 & $3067 \mathrm{C}>\mathrm{A}$ & P1023T & $\mathrm{V}$ & HEK293T/c17 & $5-\mathrm{FU}$ & Normal function & [58] \\
\hline rs114096998 & PV00861 & Exon 23 & $3067 \mathrm{C}>\mathrm{T}$ & P1023S & $\mathrm{V}$ & HEK293T/c17 & $5-\mathrm{FU}$ & Decreased function & [59] \\
\hline
\end{tabular}




\section{Dihydropyrimidinase (DHP)}

DHP, as previously mentioned, catalyzes the hydrolytic ring opening of $\mathrm{FUH}_{2}$ and $\mathrm{UH}_{2}$ and is expressed mainly in the liver and kidneys $[15,68]$. The human DHP gene (DPYS) consists of 10 exons mapped to chromosome 8q22, and features a 1560 bp open reading frame, corresponding to a 519 amino acid protein [17].

DHP deficiency is an autosomal recessive disease characterized by the accumulation of $\mathrm{UH}_{2}$ and dihydrothymine $\left(\mathrm{TH}_{2}\right)$ in blood, urine, and cerebrospinal fluid [69]. The clinical phenotype of DHP-deficient patients is highly variable, ranging from asymptomatic to exhibiting symptomatology similar to that of DPD deficiency, including seizures, intellectual disability, growth impairment, and dysmorphic facial features [70-72]. To date, 35 genetically confirmed patients with DHP deficiency have been reported [33,73-77]. However, potential asymptomatic deficiencies might be present in a population with a low frequency of DPD deficiencies. In screening 21,200 healthy Japanese infants, Sumi et al. estimated the deficiency frequency to be approximately 1/10,000 [73]. Akai et al. analyzed the DPYS coding regions from 183 Japanese individuals, in which the c.349T > C (p.W117R) and c.1001A $>$ G (p.Q334R) variants were identified with an allelic frequency of $0.27 \%$ and $1.09 \%$, respectively [78].

To date, multiple studies have reported on the relationship between DPD deficiency and the risk of developing FP-related toxicity. However, there is an increasing awareness that patients with DHP deficiencies are also prone to the development of severe FP-associated toxicity. One such study identified severe FP-related toxicity in a female breast cancer patient with the DPYS heterozygous mutation c.833G > A (p.G278D) [21]. We previously reported about a patient with severe capecitabine-associated toxicity and DHP deficiency caused by a compound DPYS heterozygous mutation, c.1001A > G (p.Q334R) and c.1393C > T (p.R465X), including a genetic analysis of the patient's family [79]. Urinary pyrimidine analysis of the patient's family revealed that the $\mathrm{UH}_{2}$ /uracil ratio of heterozygous individuals was similar to that of wild-type individuals. Although heterozygous patients are predominantly asymptomatic, severe toxicity might occur during chemotherapy containing FPs, rendering the need for genetic testing before FP administration [80].

It is noteworthy that a sizable number of DHP-deficient patients have been identified in East Asian populations. Hamajima et al. identified a single frameshift mutation and five DPYS missense variants in six Japanese patients with dihydropyrimidinuria [17]. Nakajima et al. reported two Chinese pediatric patients with DHP deficiency caused by the compound DPYS heterozygous mutation c.1001A $>$ G and c.1443 + 5G > A (exon 8 skipping) [81]. Moreover, Nakajima et al. identified eight variants, including four novel missense mutations and one novel deletion in four DHP-deficient patients [77]. Thus, DPYS polymorphisms could emerge as novel pharmacogenomic markers associated with severe FP-related toxicity in diverse global populations.

Recently, in vitro functional characterization of DHP variants using heterologous expression systems, including E. coli and mammalian cells, has been reported (Table 2). Van Kuilenburg et al. reported that in the case of 14 variants (L7V, M70T, D81G, G278D, R302Q, L337P, T343A, W360R, V364M, S379R, R412M, R465X, R475X, and R490C) expressed in E. coli, the hydrolytic ring opening of radiolabeled $\mathrm{UH}_{2}$ was markedly altered [71,76]. Hamajima et al. and Thomas et al. reported that six variants (L7V, T68R, Q334R, W360R, G435R, and R490C) showed lower activities than wild-type DHP in COS-7 and RKO cells expression systems [17,82]. We have characterized 21 DHP variants and wild-type DHP expressed in 293FT cells using $\mathrm{UH}_{2}$ and $\mathrm{FUH}_{2}$ as substrates [83]. Among these, 13 variants (N16K, T68R, M70T, D81G, G278D, R302Q, L337P, W360R, S379R, G435R, R465X, R475X, and R490C) demonstrated no enzymatic activity, and five variants (W117R, Q334R, T343A, V364M, and $\mathrm{R} 412 \mathrm{M}$ ) showed significantly lower $C L_{\text {int }}$ values than wild-type DHP. Except for L7V, the results of this study corroborated those of other in vitro studies, suggesting that the specific experimental conditions reflected the in vivo activities of the assayed variants. The divergence observed for L7V might be due to differences in assay conditions, substrate concentrations, or expression systems used. 
Table 2. DPYS variants reported in in vitro analysis.

\begin{tabular}{|c|c|c|c|c|c|c|c|}
\hline dbSNP rsID & Location & $\begin{array}{l}\text { Nucleotide } \\
\text { Change }\end{array}$ & $\begin{array}{l}\text { Amino Acid } \\
\text { Substitution }\end{array}$ & $\begin{array}{l}\text { Expression } \\
\text { System }\end{array}$ & Substrates & Effect & References \\
\hline \multirow[t]{2}{*}{ rs199618701 } & \multirow[t]{2}{*}{ Exon 1} & \multirow[t]{2}{*}{$17 \mathrm{G}>\mathrm{A}$} & \multirow[t]{2}{*}{ R6Q } & 293FT & \multirow[t]{2}{*}{$\mathrm{FUH}_{2}$} & $120 \%$ of $C L_{\text {int }}$ ratio & [83] \\
\hline & & & & 293FT & & $116 \%$ of $C L_{\text {int }}$ ratio & [83] \\
\hline \multirow[t]{2}{*}{ rs57732538 } & \multirow[t]{2}{*}{ Exon 1} & \multirow[t]{2}{*}{$19 \mathrm{C}>\mathrm{G}$} & \multirow[t]{2}{*}{ L7V } & RKO & \multirow[t]{2}{*}{$\mathrm{FUH}_{2} \mathrm{UH}_{2} \mathrm{UH}_{2}$} & $65 \%$ of wild-type DHP & [82] \\
\hline & & & & E. coli & & No function & [76] \\
\hline rs572241599 & Exon 1 & $48 \mathrm{C}>\mathrm{G}$ & N16K & 293FT & $\mathrm{FUH}_{2}$ & No function & [83] \\
\hline \multirow{2}{*}{-} & \multirow{2}{*}{ Exon 1} & \multirow{2}{*}{$203 C>G$} & \multirow{2}{*}{$\mathrm{T} 68 \mathrm{R}$} & 293FT & \multirow{2}{*}{$\mathrm{FUH}_{2} 5$-bromo- $\mathrm{UH}_{2}$} & No function & [83] \\
\hline & & & & COS-7 & & $1.5 \%$ of wild-type DHP & [17] \\
\hline \multirow{2}{*}{ rs370718225 } & \multirow{2}{*}{ Exon 1} & \multirow{2}{*}{$209 \mathrm{~T}>\mathrm{C}$} & \multirow{2}{*}{ M70T } & $293 \mathrm{FT}$ & \multirow{2}{*}{$\mathrm{FUH}_{2} \mathrm{UH}_{2}$} & No function & [83] \\
\hline & & & & E. coli & & No function & [76] \\
\hline \multirow[b]{2}{*}{-} & \multirow{2}{*}{ Exon 1} & \multirow{2}{*}{$242 \mathrm{~A}>\mathrm{G}$} & \multirow{2}{*}{$\mathrm{D} 81 \mathrm{G}$} & 293FT & \multirow{2}{*}{$\mathrm{FUH}_{2} \mathrm{UH}_{2}$} & No function & [83] \\
\hline & & & & E. coli & & No function & {$[76]$} \\
\hline- & Exon 2 & $349 \mathrm{~T}>\mathrm{C}$ & W117R & 293FT & $\mathrm{FUH}_{2}$ & $44 \%$ of $C L_{\text {int }}$ ratio & [83] \\
\hline \multirow{2}{*}{ rs36027551 } & \multirow{2}{*}{ Exon 3} & \multirow{2}{*}{$541 \mathrm{C}>\mathrm{T}$} & \multirow{2}{*}{$\mathrm{R} 181 \mathrm{~W}$} & 293FT & & $110 \%$ of $C L_{\text {int }}$ ratio & [83] \\
\hline & & & & RKO & $\mathrm{FUH}_{2} \mathrm{UH}_{2}$ & $99 \%$ of wild-type DHP & [82] \\
\hline rs751371011 & Exon 4 & $750 \mathrm{G}>\mathrm{A}$ & M250I & HEK293 & $\mathrm{UH}_{2}$ & $2 \%$ of wild-type DHP & [77] \\
\hline - & Exon 5 & $833 G>A$ & G278D & 293FT & $\mathrm{FUH}_{2} \mathrm{UH}_{2}$ & No function & [83] \\
\hline - & Exon 5 & $884 \mathrm{~A}>\mathrm{G}$ & $\mathrm{H} 295 \mathrm{R}$ & $\begin{array}{l}\text { E. coli } \\
\text { HEK293 }\end{array}$ & $\mathrm{UH}_{2}$ & $\begin{array}{l}\text { No function } \\
9.8 \% \text { of wild-type DHP }\end{array}$ & $\begin{array}{l}{[21]} \\
{[77]}\end{array}$ \\
\hline rc200013682 & Exon 5 & $905 C>4$ & $\mathrm{R} 3020$ & $293 \mathrm{FT}$ & FUHOLH & No function & [83] \\
\hline rs200913682 & Exon 5 & $905 \mathrm{G}>\mathrm{A}$ & R302Q & E. coli & $\mathrm{FUH}_{2} \mathrm{UH}_{2}$ & $3.9 \%$ of wild-type DHP & [76] \\
\hline & & & & 293FT & & $20 \%$ of $C L_{\text {int }}$ ratio & [83] \\
\hline rs121964923 & Exon 6 & $1001 \mathrm{~A}>\mathrm{G}$ & Q334R & HEK293 & $\mathrm{FUH}_{2} \mathrm{UH}_{2}$ 5-bromo- $\mathrm{UH}_{2}$ & $9.7 \%$ of wild-type DHP & [77] \\
\hline & & & & COS-7 & & $2.5 \%$ of wild-type DHP & [17] \\
\hline & & & & 293FT & & No function & [83] \\
\hline rs530911437 & Exon 6 & $1010 \mathrm{~T}>\mathrm{C}$ & L337P & E. coli & $\mathrm{FUH}_{2} \mathrm{UH}_{2}$ & No function & {$[76]$} \\
\hline & & & & $293 \mathrm{FT}$ & & $43 \%$ of $C L_{\text {int }}$ ratio & [83] \\
\hline rs201457190 & Exon 6 & $1027 \mathrm{~A}$ > G & T343A & E. coli & $\mathrm{FUH}_{2} \mathrm{UH}_{2}$ & $49 \%$ of wild-type DHP & {$[76]$} \\
\hline & & & & 293FT & & No function & [83] \\
\hline & & & & E. coli & & No function & [71] \\
\hline rs121964924 & Exon 6 & $1078 \mathrm{~T}>\mathrm{C}$ & W360R & E. coli & $\mathrm{FUH}_{2} \mathrm{UH}_{2} \mathrm{UH}_{2}$ 5-bromo-UH ${ }_{2}$ & No function & {$[76]$} \\
\hline & & & & COS-7 & & $1.2 \%$ of wild-type DHP & [17] \\
\hline rs138282507 & Exon 6 & $1090 \mathrm{G}>\mathrm{A}$ & & 293FT & & $8 \%$ of $C L_{\text {int }}$ ratio & [83] \\
\hline rs $138<8<50 /$ & Exon 6 & $1090 \mathrm{G}>\mathrm{A}$ & $\sqrt{ } 364 \mathrm{M}$ & E. coli & $\mathrm{FUH}_{2} \mathrm{UH}_{2}$ & No function & [76] \\
\hline
\end{tabular}


Table 2. Cont.

\begin{tabular}{|c|c|c|c|c|c|c|c|}
\hline dbSNP rsID & Location & $\begin{array}{l}\text { Nucleotide } \\
\text { Change }\end{array}$ & $\begin{array}{l}\text { Amino Acid } \\
\text { Substitution }\end{array}$ & $\begin{array}{l}\text { Expression } \\
\text { System }\end{array}$ & Substrates & Effect & References \\
\hline \multirow{2}{*}{ rs201258823 } & \multirow{2}{*}{ Exon 7} & \multirow{2}{*}{$1137 \mathrm{C}>\mathrm{A}$} & \multirow{2}{*}{ S379R } & 293FT & \multirow{2}{*}{$\mathrm{FUH}_{2} \mathrm{UH}_{2}$} & No function & [83] \\
\hline & & & & E. coli & & $0.20-.9 \%$ of wild-type DHP & [76] \\
\hline \multirow{2}{*}{ rs267606774 } & \multirow{2}{*}{ Exon 7} & \multirow{2}{*}{$1235 \mathrm{G}>\mathrm{T}$} & \multirow{2}{*}{$\mathrm{R} 412 \mathrm{M}$} & 293FT & \multirow{2}{*}{$\mathrm{FUH}_{2} \mathrm{UH}_{2}$} & $36 \%$ of $C L_{\text {int }}$ ratio & [83] \\
\hline & & & & E. coli & & No function & [71] \\
\hline- & Exon 8 & $1253 \mathrm{C}>\mathrm{T}$ & $\mathrm{T} 418 \mathrm{I}$ & HEK293 & $\mathrm{UH}_{2}$ & $64 \%$ of wild-type DHP & [77] \\
\hline \multirow{2}{*}{ rs267606773 } & \multirow{2}{*}{ Exon 8} & \multirow{2}{*}{$1303 \mathrm{G}>\mathrm{A}$} & \multirow{2}{*}{ G435R } & 293FT & \multirow{2}{*}{$\mathrm{FUH}_{2} 5$-bromo- $\mathrm{UH}_{2}$} & No function & [83] \\
\hline & & & & COS-7 & & $5.1 \%$ of wild-type DHP & [17] \\
\hline \multirow{2}{*}{ rs201280871 } & \multirow{2}{*}{ Exon 8} & \multirow{2}{*}{$1393 \mathrm{C}>\mathrm{T}$} & \multirow{2}{*}{$\mathrm{R} 465 \mathrm{X}$} & $293 \mathrm{FT}$ & \multirow{2}{*}{$\mathrm{FUH}_{2} \mathrm{UH}_{2}$} & No function & [83] \\
\hline & & & & E. coli & & No function & [76] \\
\hline \multirow{3}{*}{ rs61758444 } & \multirow{3}{*}{ Exon 8} & \multirow{3}{*}{$1423 \mathrm{C}>\mathrm{T}$} & \multirow{3}{*}{$\mathrm{R} 475 \mathrm{X}$} & 293FT & \multirow{3}{*}{$\mathrm{FUH}_{2} \mathrm{UH}_{2}$} & No function & [83] \\
\hline & & & & E. coli & & $0.2-0.9 \%$ of wild-type DHP & [76] \\
\hline & & & & 293FT & & No function & [83] \\
\hline \multirow[t]{2}{*}{ rs142574766 } & \multirow[t]{2}{*}{ Exon 9} & \multirow{2}{*}{$1468 \mathrm{C}>\mathrm{T}$} & \multirow[t]{2}{*}{$\mathrm{R} 490 \mathrm{C}$} & E. coli & \multirow{2}{*}{$\mathrm{FUH}_{2} \mathrm{UH}_{2} 5$-bromo- $\mathrm{UH}_{2}$} & $0.2-0.9 \%$ of wild-type DHP & [76] \\
\hline & & & & COS-7 & & $1.7 \%$ of wild-type DHP & [17] \\
\hline Rs189448963 & Exon 9 & $1469 \mathrm{G}>\mathrm{A}$ & $\mathrm{R} 490 \mathrm{H}$ & HEK293 & $\mathrm{UH}_{2}$ & $0.3 \%$ of wild-type DHP & [77] \\
\hline
\end{tabular}


Hsieh et al. reported that dimer formation is essential for DHP activity [84]. Within the cell, DHP is known to form a tetramer composed of subunits containing two zinc ions each [85-87]. Each DHP subunit consists of two domains, a large $(\beta / \alpha)_{8}$-barrel domain that binds the catalytic dimetal center and a small $\beta$-sandwich domain [88]. Each subunit also has two dynamic loops, which act as a lid for the substrate-binding pocket. DHP activity is exerted by the interaction of the C-terminus with the dynamic loop of the neighboring subunit [89-91]. We have performed immunoblotting assays of native proteins following blue native polyacrylamide gel electrophoresis and showed that oligomer formation is very important for DHP activity [83]. In the reduced or null-activity variants, the ability of DHP to form oligomers was reduced. The five variants G435R, R465X, R475X, R490C, and R490H introduce mutations in the C-terminus or lead to truncation of the C-terminus, thus affecting oligomer formation and resulting in loss of enzymatic activity. In contrast, the substitutions T68R, M70T, D81G, W117R, M250I, G278D, R302Q, Q334R, L337P, T343A, and R412M exist near the active site of the two dynamic loops, which result in conformational changes in the active site that reduce or eliminate activity. Thus, it has been clarified that changes in DHP activity are associated with amino acid substitutions, as well as changes in oligomer formation and the resulting three-dimensional structure. DHP deficiencies are rarely reported in Caucasians but are highly prevalent in Asians. Thus, we consider that these variants could serve as novel pharmacogenomic markers for the prevention of FP-related toxicity, especially in populations that have a low frequency of symptomatic DPD-deficiency cases.

\section{4. $\beta$-Ureidopropionase ( $\beta$-UP)}

$\beta$-UP catalyzes the irreversible last step, converting FUPA and $\beta$-ureidopropionic acid (bUPA) to fluoro- $\beta$-alanine and $\beta$-alanine, respectively. The human $\beta$-UP gene, UPB1, is located on chromosome 22q11, contains 10 exons, and features an $1155 \mathrm{bp}$ open reading frame; the gene encodes a polypeptide containing 384 amino acids [18]. Human $\beta$-UP activity has been detected predominantly in the liver and kidney [26,92].

$\beta$-UP deficiency is an autosomal recessive disease characterized by the accumulation of bUPA and $N$-carbamoyl- $\beta$-aminoisobutyric acid (NCBA) in urine, blood, and cerebrospinal fluid $[93,94]$. To date, 33 genetically confirmed patients with $\beta$-UP deficiency have been reported [94-100]. The clinical phenotype of these patients is highly variable but tends to center around neurological problems. Similar to DHP deficiency, $\beta$-UP deficiency is often reported in East Asian populations, including Japan and China. Although it has been reported that severe FP-related toxicity is caused by DPD and DHP deficiencies, little is known about the relationship between $\beta$-UP deficiency and FP-related toxicity.

There have been several reports of the in vitro analysis of 13 UPB1 variants with amino acid substitutions identified in $\beta$-UP-deficient patients (Table 3). Van Kuilenburg et al. and Thomas et al. reported that variant A85E expressed in E. coli and RKO cells was inactive [93,101]. In a separate study, van Kuilenburg et al., using an E. coli expression system, analyzed six $\beta$-UP variants (L13S, G235R, R236W, S264R, R326Q, and T359M) that had been previously identified in $16 \beta$-UP-deficient patients, showing a significant reduction or loss of activity in all of them [95]. Nakajima et al. reported that the G31S, E271K, and R326Q variants expressed in HEK293 cells showed profound reductions in activity [97]. Moreover, Nakajima et al. performed native polyacrylamide gel electrophoresis of $\beta$-UP expressed in HEK293 cells and showed that octamer formation is necessary for $\beta$-UP activity as well as DHP activity. The majority of variants showed a significant reduction in enzymatic activity. However, whether these variants contribute to the development of FP-related toxicity remains unclear. 
Table 3. UPB1 variants identified in $\beta$-UP deficient patients.

\begin{tabular}{|c|c|c|c|c|c|c|c|}
\hline db SNP rsID & Location & $\begin{array}{l}\text { Nucleotide } \\
\text { Change }\end{array}$ & $\begin{array}{l}\text { Amino Acid } \\
\text { Substitution }\end{array}$ & $\begin{array}{l}\text { Expression } \\
\text { System }\end{array}$ & Substrates & Effect & References \\
\hline- & Exon 1 & c.38T >C & p.L13S & E. coli & bUPA & $6 \%$ of wild-type $\beta$-UP & [95] \\
\hline rs200145797 & Exon 1 & c. $91 \mathrm{G}>\mathrm{A}$ & p.G31S & HEK293 & bUPA & $52 \%$ of wild-type $\beta$-UP & [97] \\
\hline rs121908066 & Exon 2 & c. $209 \mathrm{G}>\mathrm{C}$ & p.R70P & \multicolumn{3}{|c|}{ No reports of in vitro study } & [98] \\
\hline & & & & E. coli & bUPA & No function & [93] \\
\hline rs34035085 & Exon 2 & c. $254 \mathrm{C}>\mathrm{A}$ & p.A85E & RKO & bUPA & $2.7 \%$ of wild-type $\beta$-UP & [101] \\
\hline- & Exon 6 & c.703G > A & p.G235R & E. coli & bUPA & No function & [95] \\
\hline rs144135211 & Exon 6 & c. $706 \mathrm{C}>\mathrm{T}$ & p.R236W & E. coli & bUPA & No function & [95] \\
\hline rs145766755 & Exon 7 & c. $792 \mathrm{C}>\mathrm{A}$ & p.S264R & E. coli & bUPA & $20 \%$ of wild-type $\beta$-UP & [95] \\
\hline- & Exon 7 & c. $811 \mathrm{G}>\mathrm{A}$ & p.E271K & HEK293 & bUPA & $0.7 \%$ of wild-type $\beta$-UP & [97] \\
\hline - & Exon 7 & c. $851 G>T$ & p.C284F & \multicolumn{3}{|c|}{ No reports of in vitro study } & [99] \\
\hline rs1375840064 & Exon 7 & c. $853 \mathrm{G}>\mathrm{A}$ & p.A285T & \multicolumn{3}{|c|}{ No reports of in vitro study } & [99] \\
\hline- & Exon 7 & c.857T > C & p.I286T & HEK293 & bUPA & $70 \%$ of wild-type $\beta$-UP & [97] \\
\hline & & & & E. coli & bUPA & No function & [95] \\
\hline rs1 & E) & c. 97 & p.R326Q & HEK293 & bUPA & $1.3 \%$ of wild-type $\beta$-UP & {$[97]$} \\
\hline rs369879221 & Exon 10 & c. $1076 \mathrm{C}>\mathrm{T}$ & p.T359M & E. coli & bUPA & No function & [95] \\
\hline
\end{tabular}


Fidlerova et al. performed an analysis of the entire UPB1 coding sequence from 113 Czech cancer patients treated using FP-based chemotherapy [102]. Nine UPB1 variants were detected in a subpopulation of patients exhibiting severe toxicity, including a novel mutation affecting the coding sequence. An analysis of the effect of UPB1 variants on FP-related toxicity in the population of all analyzed patients revealed an association between the c. $-80 \mathrm{C}>\mathrm{G}$ (rs2070474) variant and gastrointestinal toxicity. In addition, a strong positive correlation was found between carriers of the homozygous c. $-80 \mathrm{G}$ variant and the development of severe mucositis. Thomas et al. deduced that the c. $-80 \mathrm{G}$ variants might alter the potential binding sites of transcription factors, resulting in a statistically non-significant decrease in UPB1 gene expression in patients who are homozygous for the c.-80G allele. This indicates the possibility that $U P B 1$ variants have an additive and relatively minor effect on the development of FP-related toxicity compared with that of the DPYD and DPYS variants.

\section{Other Considerations}

Genetic variations in TYMS, MTHFR, and miR-27a have also been associated with FP-related toxicity. Clinical and preclinical studies have shown the importance of intracellular levels of TYMS, a target for 5-FU involved in DNA repair and synthesis [103], as a determinant of sensitivity to 5-FU treatment. Its overexpression stemming from polymorphic TYMS variations lead to differing response rates to 5-FU therapy [104]. The three most studied TYMS genetic polymorphisms are the variable numbers of tandem repeat (VNTR) polymorphisms comprising 28 bp sequence repeats (rs34743033), rs2853542C > G, and the $3^{\prime}$-untranslated region polymorphism 1494delTTAAAG (rs34489327). These polymorphisms alter gene expression, mRNA stability, or TYMS expression levels, resulting in the development of treatment resistance and toxicity [105-107]. MTHFR plays a role in the metabolism of folate and forms the reduced folate cofactor essential for TYMS inhibition by 5-FU. Two non-synonymous variants, c.677C > T (p.A222V, rs1801133) and c.1298A > C (p.E429A, rs1801131), alter intracellular folate distribution and decrease enzymatic activity $[105,107]$. The micro RNA miR-27a polymorphism (rs895819A > G) has been associated with FP-related toxicity, more so in DPD-deficient patients, as increased miR-27a expression leads to decreased DPD mRNA expression [108-110]. To date, however, studies involving these genetic polymorphisms have yielded inconsistent results, and further assessment is needed to assess their clinical utility and potential use as biomarkers.

\section{Conclusions}

FPs are degraded by three metabolic enzymes (DPD, DHP, and $\beta$-UP), and a reduction or elimination of their activities leads to severe FP-related toxicity. Therefore, predicting enzymatic activity is critical before the administration of FPs, in which in vitro testing has proven to be a useful complementary method to in vivo testing. This review summarized the findings on the functional characterization of DPD, DHP, and $\beta$-UP using in vitro analysis. To date, a large number of DPD variants have been analyzed, giving rise to a significant body of evidence regarding the four most commonly identified risk variants in Caucasians $\left(D P Y D^{*} 2 A, D P Y D^{*} 13\right.$, c.1129 - 5923C > G/hapB3, and c.2846A $>$ T) that are associated with an increased risk of 5-FU-related toxicity. Additionally, a system for predicting in vivo DPD activity has been developed on the basis of in vitro analysis results. This has provided further evidence that rare DHP variants might be useful predictive biomarkers of FP-related toxicity in populations with low frequencies of DPD deficiency, as is the case for Asians. Notably, $\beta$-UP is not known to be associated with FP-related toxicity, although variants with reduced function have been identified. Currently, studies comprising in vivo and in vitro correlation of frequent $D P Y D$ polymorphisms are advancing applicability as well as underlying the importance of including infrequent DPYD, DPYS, and UPB1 variants, as their collective data is insufficient to establish their clinical consequences fully. Additional in vitro and large-scale in vivo studies using standardized methodologies are needed to generate clear evidence for rare variants and verify existing associative studies. 
Recently, the underlying mechanisms by which amino acid substitutions alter enzymatic activities by influencing three-dimensional structures have been elucidated; these findings have significant implications toward the interpretation of previously acquired data and how they could be further used to aid clinical decision making for optimal treatments and forewarning the need for alternative chemotherapy regimens. We expect that this report and others related to genetic FP-metabolizing enzyme variants will be useful in the development and further validation of pharmacogenetic testing with the future inclusion of additional biomarkers. In this way, these developments could lead to optimal personalized medicine grounded on genetic polymorphisms.

Author Contributions: Conceptualization, E.H. and M.H.; writing—original draft preparation, E.H.; writing一review and editing, E.H., E.G.R., and M.H. All authors have read and agreed to the published version of the manuscript.

Funding: This work was supported by grants from the Japan Agency for Medical Research and Development, AMED (under grant number 20kk0305009h0002).

Conflicts of Interest: The authors declare no conflict of interest.

\section{References}

1. Pan, X.; Wang, C.; Wang, F.; Li, P.; Hu, Z.; Shan, Y.; Zhang, J. Development of 5-Fluorouracil derivatives as anticancer agents. Curr. Med. Chem. 2011, 18, 4538-4556. [CrossRef] [PubMed]

2. Kilic, L.; Ordu, C.; Yildiz, I.; Sen, F.; Keskin, S.; Ciftci, R.; Pilanci, K.N. Current adjuvant treatment modalities for gastric cancer: From history to the future. World J. Gastrointest. Oncol. 2016, 8, 439-449. [CrossRef] [PubMed]

3. Lucas, A.S.; O'Neil, B.H.; Goldberg, R.M. A decade of advances in cytotoxic chemotherapy for metastatic colorectal cancer. Clin. Colorectal. Cancer 2011, 10, 238-244. [CrossRef] [PubMed]

4. Yap, Y.S.; Kwok, L.L.; Syn, N.; Chay, W.Y.; Chia, J.W.K.; Tham, C.K.; Wong, N.S.; Lo, S.K.; Dent, R.A.; Tan, S.; et al. Predictors of Hand-Foot Syndrome and Pyridoxine for Prevention of Capecitabine-Induced Hand-Foot Syndrome: A Randomized Clinical Trial. JAMA Oncol. 2017, 3, 1538-1545. [CrossRef]

5. Lamberti, M.; Porto, S.; Zappavigna, S.; Addeo, E.; Marra, M.; Miraglia, N.; Sannolo, N.; Vanacore, D.; Stiuso, P.; Caraglia, M. A mechanistic study on the cardiotoxicity of 5-fluorouracil in vitro and clinical and occupational perspectives. Toxicol. Lett. 2014, 227, 151-156. [CrossRef] [PubMed]

6. Kim, Y.A.; Chung, H.C.; Choi, H.J.; Rha, S.Y.; Seong, J.S.; Jeung, H.C. Intermediate dose 5-fluorouracil-induced encephalopathy. Jpn. J. Clin. Oncol. 2006, 36, 55-59. [CrossRef] [PubMed]

7. Twelves, C.; Wong, A.; Nowacki, M.P.; Abt, M.; Burris, H., 3rd; Carrato, A.; Cassidy, J.; Cervantes, A.; Fagerberg, J.; Georgoulias, V.; et al. Capecitabine as adjuvant treatment for stage III colon cancer. N. Engl. J. Med. 2005, 352, 2696-2704. [CrossRef]

8. Saltz, L.B.; Niedzwiecki, D.; Hollis, D.; Goldberg, R.M.; Hantel, A.; Thomas, J.P.; Fields, A.L.; Mayer, R.J. Irinotecan fluorouracil plus leucovorin is not superior to fluorouracil plus leucovorin alone as adjuvant treatment for stage III colon cancer: Results of CALGB 89803. J. Clin. Oncol. Off. J. Am. Soc. Clin. Oncol. 2007, 25, 3456-3461. [CrossRef]

9. Lam, S.W.; Guchelaar, H.J.; Boven, E. The role of pharmacogenetics in capecitabine efficacy and toxicity. Cancer Treat. Rev. 2016, 50, 9-22. [CrossRef]

10. Kunicka, T.; Prochazka, P.; Krus, I.; Bendova, P.; Protivova, M.; Susova, S.; Hlavac, V.; Liska, V.; Novak, P.; Schneiderova, M.; et al. Molecular profile of 5-fluorouracil pathway genes in colorectal carcinoma. BMC Cancer 2016, 16, 795. [CrossRef]

11. Kim, J.Y.; Shin, E.; Kim, J.W.; Lee, H.S.; Lee, D.W.; Kim, S.H.; Lee, J.O.; Kim, Y.J.; Kim, J.H.; Bang, S.M.; et al. Impact of intratumoral expression levels of fluoropyrimidine-metabolizing enzymes on treatment outcomes of adjuvant S-1 therapy in gastric cancer. PLoS ONE 2015, 10, e0120324. [CrossRef] [PubMed]

12. Daher, G.C.; Harris, B.E.; Diasio, R.B. Metabolism of pyrimidine analogues and their nucleosides. Pharmacol. Ther. 1990, 48, 189-222. [CrossRef]

13. Heggie, G.D.; Sommadossi, J.P.; Cross, D.S.; Huster, W.J.; Diasio, R.B. Clinical pharmacokinetics of 5-fluorouracil and its metabolites in plasma, urine, and bile. Cancer Res. 1987, 47, 2203-2206. [PubMed] 
14. Porter, D.J.; Harrington, J.A.; Almond, M.R.; Lowen, G.T.; Spector, T. (R)-5-fluoro-5,6-dihydrouracil: Kinetics of oxidation by dihydropyrimidine dehydrogenase and hydrolysis by dihydropyrimidine aminohydrolase. Biochem. Pharmacol. 1994, 48, 775-779. [CrossRef]

15. Kikugawa, M.; Kaneko, M.; Fujimoto-Sakata, S.; Maeda, M.; Kawasaki, K.; Takagi, T.; Tamaki, N. Purification, characterization and inhibition of dihydropyrimidinase from rat liver. Eur. J. Biochem. 1994, 219, 393-399. [CrossRef]

16. Lu, Z.H.; Zhang, R.; Diasio, R.B. Purification and characterization of dihydropyrimidine dehydrogenase from human liver. J. Biol. Chem. 1992, 267, 17102-17109.

17. Hamajima, N.; Kouwaki, M.; Vreken, P.; Matsuda, K.; Sumi, S.; Imaeda, M.; Ohba, S.; Kidouchi, K.; Nonaka, M.; Sasaki, M.; et al. Dihydropyrimidinase deficiency: Structural organization, chromosomal localization, and mutation analysis of the human dihydropyrimidinase gene. Am. J. Hum. Genet. 1998, 63, 717-726. [CrossRef]

18. Vreken, P.; van Kuilenburg, A.B.; Hamajima, N.; Meinsma, R.; van Lenthe, H.; Gohlich-Ratmann, G.; Assmann, B.E.; Wevers, R.A.; van Gennip, A.H. cDNA cloning, genomic structure and chromosomal localization of the human BUP-1 gene encoding beta-ureidopropionase. Biochim. Biophys. Acta 1999, 1447, 251-257. [CrossRef]

19. Amstutz, U.; Farese, S.; Aebi, S.; Largiader, C.R. Dihydropyrimidine dehydrogenase gene variation and severe 5-fluorouracil toxicity: A haplotype assessment. Pharmacogenomics 2009, 10, 931-944. [CrossRef]

20. van Kuilenburg, A.B.P.; Haasjes, J.; Richel, D.J.; Zoetekouw, L.; Van Lenthe, H.; De Abreu, R.A.; Maring, J.G.; Vreken, P.; van Gennip, A.H. Clinical Implications of Dihydropyrimidine Dehydrogenase (DPD) Deficiency in Patients with Severe 5-Fluorouracil-associated Toxicity: Identification of New Mutations in the DPD Gene. Clin. Cancer Res. 2000, 6, 4705-4712.

21. van Kuilenburg, A.B.; Meinsma, R.; Zonnenberg, B.A.; Zoetekouw, L.; Baas, F.; Matsuda, K.; Tamaki, N.; van Gennip, A.H. Dihydropyrimidinase deficiency and severe 5-fluorouracil toxicity. Clin. Cancer Res. Off. J. Am. Assoc. Cancer Res. 2003, 9, 4363-4367.

22. Amstutz, U.; Froehlich, T.K.; Largiader, C.R. Dihydropyrimidine dehydrogenase gene as a major predictor of severe 5-fluorouracil toxicity. Pharmacogenomics 2011, 12, 1321-1336. [CrossRef] [PubMed]

23. Meulendijks, D.; Cats, A.; Beijnen, J.H.; Schellens, J.H. Improving safety of fluoropyrimidine chemotherapy by individualizing treatment based on dihydropyrimidine dehydrogenase activity-Ready for clinical practice? Cancer Treat. Rev. 2016, 50, 23-34. [CrossRef] [PubMed]

24. Sistonen, J.; Buchel, B.; Froehlich, T.K.; Kummer, D.; Fontana, S.; Joerger, M.; van Kuilenburg, A.B.; Largiader, C.R. Predicting 5-fluorouracil toxicity: DPD genotype and 5,6-dihydrouracil:uracil ratio. Pharmacogenomics 2014, 15, 1653-1666. [CrossRef] [PubMed]

25. Hiratsuka, M. In vitro assessment of the allelic variants of cytochrome P450. Drug Metab. Pharmacokinet. 2012, 27, 68-84. [CrossRef] [PubMed]

26. van Kuilenburg, A.B.; van Lenthe, H.; van Gennip, A.H. Activity of pyrimidine degradation enzymes in normal tissues. Nucleosides Nucleotides Nucleic Acids 2006, 25, 1211-1214. [CrossRef]

27. Wei, X.; Elizondo, G.; Sapone, A.; McLeod, H.L.; Raunio, H.; Fernandez-Salguero, P.; Gonzalez, F.J. Characterization of the human dihydropyrimidine dehydrogenase gene. Genomics 1998, 51, 391-400. [CrossRef]

28. Bakkeren, J.A.; De Abreu, R.A.; Sengers, R.C.; Gabreels, F.J.; Maas, J.M.; Renier, W.O. Elevated urine, blood and cerebrospinal fluid levels of uracil and thymine in a child with dihydrothymine dehydrogenase deficiency. Clin. Chim. Acta Int. J. Clin. Chem. 1984, 140, 247-256. [CrossRef]

29. Berger, R.; Stoker-de Vries, S.A.; Wadman, S.K.; Duran, M.; Beemer, F.A.; de Bree, P.K.; Weits-Binnerts, J.J.; Penders, T.J.; van der Woude, J.K. Dihydropyrimidine dehydrogenase deficiency leading to thymine-uraciluria. An inborn error of pyrimidine metabolism. Clin. Chim. Acta Int. J. Clin. Chem. 1984, 141, 227-234. [CrossRef]

30. van Kuilenburg, A.B. Dihydropyrimidine dehydrogenase and the efficacy and toxicity of 5-fluorouracil. Eur. J. Cancer 2004, 40, 939-950. [CrossRef]

31. Al-Sanna'a, N.A.; Van Kuilenburg, A.B.; Atrak, T.M.; Abdul-Jabbar, M.A.; Van Gennip, A.H. Dihydropyrimidine dehydrogenase deficiency presenting at birth. J. Inherit. Metab. Dis. 2005, 28, 793-796. [CrossRef] [PubMed]

32. Boisdron-Celle, M.; Remaud, G.; Traore, S.; Poirier, A.L.; Gamelin, L.; Morel, A.; Gamelin, E. 5-Fluorouracil-related severe toxicity: A comparison of different methods for the pretherapeutic detection of dihydropyrimidine dehydrogenase deficiency. Cancer Lett. 2007, 249, 271-282. [CrossRef] [PubMed] 
33. Hayashi, K.; Kidouchi, K.; Sumi, S.; Mizokami, M.; Orito, E.; Kumada, K.; Ueda, R.; Wada, Y. Possible prediction of adverse reactions to pyrimidine chemotherapy from urinary pyrimidine levels and a case of asymptomatic adult dihydropyrimidinuria. Clin. Cancer Res. Off. J. Am. Assoc. Cancer Res. 1996, 2, 1937-1941.

34. Ezzeldin, H.; Diasio, R. Dihydropyrimidine dehydrogenase deficiency, a pharmacogenetic syndrome associated with potentially life-threatening toxicity following 5-fluorouracil administration. Clin. Colorectal. Cancer 2004, 4, 181-189. [CrossRef]

35. Mounier-Boutoille, H.; Boisdron-Celle, M.; Cauchin, E.; Galmiche, J.P.; Morel, A.; Gamelin, E.; Matysiak-Budnik, T. Lethal outcome of 5-fluorouracil infusion in a patient with a total DPD deficiency and a double DPYD and UTG1A1 gene mutation. Br. J. Clin. Pharmacol. 2010, 70, 280-283. [CrossRef]

36. Sahu, A.; Ramaswamy, A.; Ostwal, V. Dihydro pyrimidine dehydrogenase deficiency in patients treated with capecitabine based regimens: A tertiary care centre experience. J. Gastrointest. Oncol. 2016, 7, 380-386. [CrossRef]

37. Sistonen, J.; Smith, C.; Fu, Y.K.; Largiader, C.R. A new DPYD genotyping assay for improving the safety of 5-fluorouracil therapy. Clin. Chim. Acta Int. J. Clin. Chem. 2012, 414, 109-111. [CrossRef]

38. Iyer, S.N.; Singhal, R.S.; Hegde, M.R.; Ankala, A. Genetic variation in dihydropyrimidine dehydrogenase (DPYD) gene in a healthy adult Indian population. Ann. Hum. Biol. 2015, 42, 97-100. [CrossRef]

39. Vaudo, C.E.; Gil, B.; Galuski, K.; Zarwan, C.; Nugent, F.W. Early-Onset 5-Fluorouracil Toxicity in a Patient Negative for Dihydropyrimidine Dehydrogenase Mutations: The Clinical Course of Reversal with Uridine Triacetate. Pharmacotherapy 2016, 36, e178-e182. [CrossRef]

40. Thomas, F.; Hennebelle, I.; Delmas, C.; Lochon, I.; Dhelens, C.; Garnier Tixidre, C.; Bonadona, A.; Penel, N.; Goncalves, A.; Delord, J.P.; et al. Genotyping of a family with a novel deleterious DPYD mutation supports the pretherapeutic screening of DPD deficiency with dihydrouracil/uracil ratio. Clin. Pharmacol. Ther. 2016, 99, 235-242. [CrossRef]

41. Henricks, L.M.; Siemerink, E.J.M.; Rosing, H.; Meijer, J.; Goorden, S.M.I.; Polstra, A.M.; Zoetekouw, L.; Cats, A.; Schellens, J.H.M.; van Kuilenburg, A.B.P. Capecitabine-based treatment of a patient with a novel DPYD genotype and complete dihydropyrimidine dehydrogenase deficiency. Int. J. Cancer 2018, 142, 424-430. [CrossRef]

42. Gross, E.; Meul, C.; Raab, S.; Propping, C.; Avril, S.; Aubele, M.; Gkazepis, A.; Schuster, T.; Grebenchtchikov, N.; Schmitt, M.; et al. Somatic copy number changes in DPYD are associated with lower risk of recurrence in triple-negative breast cancers. Br. J. Cancer 2013, 109, 2347-2355. [CrossRef]

43. van Kuilenburg, A.B.; Meijer, J.; Mul, A.N.; Meinsma, R.; Schmid, V.; Dobritzsch, D.; Hennekam, R.C.; Mannens, M.M.; Kiechle, M.; Etienne-Grimaldi, M.C.; et al. Intragenic deletions and a deep intronic mutation affecting pre-mRNA splicing in the dihydropyrimidine dehydrogenase gene as novel mechanisms causing 5-fluorouracil toxicity. Hum. Genet. 2010, 128, 529-538. [CrossRef]

44. Qin, F.; Zhang, H.; Huang, Y.; Yang, L.; Yu, F.; Liu, X.; Fu, L.; Gu, F.; Ma, Y. Effect of dihydropyrimidine dehydrogenase single nucleotide polymorphisms on prognosis of breast cancer patients with chemotherapy. Oncotarget 2017, 8, 112060-112075. [CrossRef] [PubMed]

45. Seck, K.; Riemer, S.; Kates, R.; Ullrich, T.; Lutz, V.; Harbeck, N.; Schmitt, M.; Kiechle, M.; Diasio, R.; Gross, E. Analysis of the DPYD gene implicated in 5-fluorouracil catabolism in a cohort of Caucasian individuals. Clin. Cancer Res. Off. J. Am. Assoc. Cancer Res. 2005, 11, 5886-5892. [CrossRef]

46. Amstutz, U.; Henricks, L.M.; Offer, S.M.; Barbarino, J.; Schellens, J.H.M.; Swen, J.J.; Klein, T.E.; McLeod, H.L.; Caudle, K.E.; Diasio, R.B.; et al. Clinical Pharmacogenetics Implementation Consortium (CPIC) Guideline for Dihydropyrimidine Dehydrogenase Genotype and Fluoropyrimidine Dosing: 2017 Update. Clin. Pharmacol. Ther. 2018, 103, 210-216. [CrossRef] [PubMed]

47. Lunenburg, C.; van der Wouden, C.H.; Nijenhuis, M.; Crommentuijn-van Rhenen, M.H.; de Boer-Veger, N.J.; Buunk, A.M.; Houwink, E.J.F.; Mulder, H.; Rongen, G.A.; van Schaik, R.H.N.; et al. Dutch Pharmacogenetics Working Group (DPWG) guideline for the gene-drug interaction of DPYD and fluoropyrimidines. Eur. J. Hum. Genet. 2020, 28, 508-517. [CrossRef] [PubMed]

48. Shin, J.G.; Cheong, H.S.; Kim, J.Y.; Kim, L.H.; Han, C.S.; Kim, J.O.; Kim, H.D.; Kim, Y.H.; Chung, M.W.; Han, S.Y.; et al. Screening of dihydropyrimidine dehydrogenase genetic variants by direct sequencing in different ethnic groups. J. Korean Med. Sci. 2013, 28, 1129-1133. [CrossRef] [PubMed] 
49. Maekawa, K.; Saeki, M.; Saito, Y.; Ozawa, S.; Kurose, K.; Kaniwa, N.; Kawamoto, M.; Kamatani, N.; Kato, K.; Hamaguchi, T.; et al. Genetic variations and haplotype structures of the DPYD gene encoding dihydropyrimidine dehydrogenase in Japanese and their ethnic differences. J. Hum. Genet. 2007, 52, 804-819. [CrossRef]

50. He, Y.F.; Wei, W.; Zhang, X.; Li, Y.H.; Li, S.; Wang, F.H.; Lin, X.B.; Li, Z.M.; Zhang, D.S.; Huang, H.Q.; et al. Analysis of the DPYD gene implicated in 5-fluorouracil catabolism in Chinese cancer patients. J. Clin. Pharm. Ther. 2008, 33, 307-314. [CrossRef]

51. Lunenburg, C.; Henricks, L.M.; Guchelaar, H.J.; Swen, J.J.; Deenen, M.J.; Schellens, J.H.M.; Gelderblom, H. Prospective DPYD genotyping to reduce the risk of fluoropyrimidine-induced severe toxicity: Ready for prime time. Eur. J. Cancer 2016, 54, 40-48. [CrossRef] [PubMed]

52. Takimoto, C.H.; Lu, Z.H.; Zhang, R.; Liang, M.D.; Larson, L.V.; Cantilena, L.R., Jr.; Grem, J.L.; Allegra, C.J.; Diasio, R.B.; Chu, E. Severe neurotoxicity following 5-fluorouracil-based chemotherapy in a patient with dihydropyrimidine dehydrogenase deficiency. Clin. Cancer Res. Off. J. Am. Assoc. Cancer Res. 1996, 2, 477-481.

53. Lu, Z.; Zhang, R.; Diasio, R.B. Dihydropyrimidine dehydrogenase activity in human peripheral blood mononuclear cells and liver: Population characteristics, newly identified deficient patients, and clinical implication in 5-fluorouracil chemotherapy. Cancer Res. 1993, 53, 5433-5438. [PubMed]

54. Offer, S.M.; Wegner, N.J.; Fossum, C.; Wang, K.; Diasio, R.B. Phenotypic profiling of DPYD variations relevant to 5-fluorouracil sensitivity using real-time cellular analysis and in vitro measurement of enzyme activity. Cancer Res. 2013, 73, 1958-1968. [CrossRef] [PubMed]

55. Kuilenburg, A.; Meijer, J.; Tanck, M.W.T.; Dobritzsch, D.; Zoetekouw, L.; Dekkers, L.L.; Roelofsen, J.; Meinsma, R.; Wymenga, M.; Kulik, W.; et al. Phenotypic and clinical implications of variants in the dihydropyrimidine dehydrogenase gene. Biochim. Biophys. Acta 2016, 1862, 754-762. [CrossRef]

56. van Kuilenburg, A.B.; Meijer, J.; Maurer, D.; Dobritzsch, D.; Meinsma, R.; Los, M.; Knegt, L.C.; Zoetekouw, L.; Jansen, R.L.; Dezentje, V.; et al. Severe fluoropyrimidine toxicity due to novel and rare DPYD missense mutations, deletion and genomic amplification affecting DPD activity and mRNA splicing. Biochim. Biophys. Acta Mol. Basis Dis. 2017, 1863, 721-730. [CrossRef]

57. Ogura, K.; Ohnuma, T.; Minamide, Y.; Mizuno, A.; Nishiyama, T.; Nagashima, S.; Kanamaru, M.; Hiratsuka, A.; Watabe, T.; Uematsu, T. Dihydropyrimidine dehydrogenase activity in 150 healthy Japanese volunteers and identification of novel mutations. Clin. Cancer Res. Off. J. Am. Assoc. Cancer Res. 2005, 11, 5104-5111. [CrossRef]

58. Offer, S.M.; Fossum, C.C.; Wegner, N.J.; Stuflesser, A.J.; Butterfield, G.L.; Diasio, R.B. Comparative functional analysis of DPYD variants of potential clinical relevance to dihydropyrimidine dehydrogenase activity. Cancer Res. 2014, 74, 2545-2554. [CrossRef]

59. Elraiyah, T.; Jerde, C.R.; Shrestha, S.; Wu, R.; Nie, Q.; Giama, N.H.; Sarangi, V.; Roberts, L.R.; Offer, S.M.; Diasio, R.B. Novel Deleterious Dihydropyrimidine Dehydrogenase Variants May Contribute to 5-Fluorouracil Sensitivity in an East African Population. Clin. Pharmacol. Ther. 2017, 101, 382-390. [CrossRef]

60. Hishinuma, E.; Narita, Y.; Saito, S.; Maekawa, M.; Akai, F.; Nakanishi, Y.; Yasuda, J.; Nagasaki, M.; Yamamoto, M.; Yamaguchi, H.; et al. Functional Characterization of 21 Allelic Variants of Dihydropyrimidine Dehydrogenase Identified in 1070 Japanese Individuals. Drug Metab. Dispos. Biol. Fate Chem. 2018, 46, 1083-1090. [CrossRef]

61. Schnackerz, K.D.; Dobritzsch, D.; Lindqvist, Y.; Cook, P.F. Dihydropyrimidine dehydrogenase: A flavoprotein with four iron-sulfur clusters. Biochim. Biophys. Acta 2004, 1701, 61-74. [CrossRef] [PubMed]

62. Dobritzsch, D.; Ricagno, S.; Schneider, G.; Schnackerz, K.D.; Lindqvist, Y. Crystal structure of the productive ternary complex of dihydropyrimidine dehydrogenase with NADPH and 5-iodouracil. Implications for mechanism of inhibition and electron transfer. J. Biol. Chem. 2002, 277, 13155-13166. [CrossRef] [PubMed]

63. Dobritzsch, D.; Schneider, G.; Schnackerz, K.D.; Lindqvist, Y. Crystal structure of dihydropyrimidine dehydrogenase, a major determinant of the pharmacokinetics of the anti-cancer drug 5-fluorouracil. EMBO J. 2001, 20, 650-660. [CrossRef] [PubMed]

64. Mattison, L.K.; Johnson, M.R.; Diasio, R.B. A comparative analysis of translated dihydropyrimidine dehydrogenase cDNA; conservation of functional domains and relevance to genetic polymorphisms. Pharmacogenetics 2002, 12, 133-144. [CrossRef] 
65. Lohkamp, B.; Voevodskaya, N.; Lindqvist, Y.; Dobritzsch, D. Insights into the mechanism of dihydropyrimidine dehydrogenase from site-directed mutagenesis targeting the active site loop and redox cofactor coordination. Biochim. Biophys. Acta 2010, 1804, 2198-2206. [CrossRef]

66. Henricks, L.M.; Lunenburg, C.A.; Meulendijks, D.; Gelderblom, H.; Cats, A.; Swen, J.J.; Schellens, J.H.; Guchelaar, H.J. Translating DPYD genotype into DPD phenotype: Using the DPYD gene activity score. Pharmacogenomics 2015, 16, 1277-1286. [CrossRef]

67. Shrestha, S.; Zhang, C.; Jerde, C.R.; Nie, Q.; Li, H.; Offer, S.M.; Diasio, R.B. Gene-Specific Variant Classifier (DPYD-Varifier) to Identify Deleterious Alleles of Dihydropyrimidine Dehydrogenase. Clin. Pharmacol. Ther. 2018, 104, 709-718. [CrossRef]

68. Hamajima, N.; Matsuda, K.; Sakata, S.; Tamaki, N.; Sasaki, M.; Nonaka, M. A novel gene family defined by human dihydropyrimidinase and three related proteins with differential tissue distribution. Gene 1996, 180, 157-163. [CrossRef]

69. Duran, M.; Rovers, P.; de Bree, P.K.; Schreuder, C.H.; Beukenhorst, H.; Dorland, L.; Berger, R. Dihydropyrimidinuria: A new inborn error of pyrimidine metabolism. J. Inherit. Metab. Dis. 1991, 14, 367-370. [CrossRef]

70. Ohba, S.; Kidouchi, K.; Sumi, S.; Imaeda, M.; Takeda, N.; Yoshizumi, H.; Tatematsu, A.; Kodama, K.; Yamanaka, K.; Kobayashi, M.; et al. Dihydropyrimidinuria: The first case in Japan. Adv. Exp. Med. Biol. 1994, 370, 383-386. [CrossRef]

71. van Kuilenburg, A.B.; Meijer, J.; Dobritzsch, D.; Meinsma, R.; Duran, M.; Lohkamp, B.; Zoetekouw, L.; Abeling, N.G.; van Tinteren, H.L.; Bosch, A.M. Clinical, biochemical and genetic findings in two siblings with a dihydropyrimidinase deficiency. Mol. Genet. Metab. 2007, 91, 157-164. [CrossRef] [PubMed]

72. Yeung, C.W.; Yau, M.M.; Ma, C.K.; Siu, T.S.; Tam, S.; Lam, C.W. Diagnosis of dihydropyrimidinase deficiency in a Chinese boy with dihydropyrimidinuria. Hong Kong Med. J./Xianggang Yi Xue Za Zhi 2013, 19, $272-275$. [CrossRef]

73. Sumi, S.; Imaeda, M.; Kidouchi, K.; Ohba, S.; Hamajima, N.; Kodama, K.; Togari, H.; Wada, Y. Population and family studies of dihydropyrimidinuria: Prevalence, inheritance mode, and risk of fluorouracil toxicity. Am. J. Med Genet. 1998, 78, 336-340. [CrossRef]

74. van Gennip, A.H.; Abeling, N.G.; Stroomer, A.E.; van Lenthe, H.; Bakker, H.D. Clinical and biochemical findings in six patients with pyrimidine degradation defects. J. Inherit. Metab. Dis. 1994, 17, 130-132. [CrossRef] [PubMed]

75. van Gennip, A.H.; de Abreu, R.A.; van Lenthe, H.; Bakkeren, J.; Rotteveel, J.; Vreken, P.; van Kuilenburg, A.B. Dihydropyrimidinase deficiency: Confirmation of the enzyme defect in dihydropyrimidinuria. J. Inherit. Metab. Dis. 1997, 20, 339-342. [CrossRef] [PubMed]

76. van Kuilenburg, A.B.; Dobritzsch, D.; Meijer, J.; Meinsma, R.; Benoist, J.F.; Assmann, B.; Schubert, S.; Hoffmann, G.F.; Duran, M.; de Vries, M.C.; et al. Dihydropyrimidinase deficiency: Phenotype, genotype and structural consequences in 17 patients. Biochim. Biophys. Acta 2010, 1802, 639-648. [CrossRef]

77. Nakajima, Y.; Meijer, J.; Dobritzsch, D.; Ito, T.; Zhang, C.; Wang, X.; Watanabe, Y.; Tashiro, K.; Meinsma, R.; Roelofsen, J.; et al. Dihydropyrimidinase deficiency in four East Asian patients due to novel and rare DPYS mutations affecting protein structural integrity and catalytic activity. Mol. Genet. Metab. 2017, 122, $216-222$. [CrossRef]

78. Akai, F.; Hosono, H.; Hirasawa, N.; Hiratsuka, M. Novel single nucleotide polymorphisms of the dihydropyrimidinase gene (DPYS) in Japanese individuals. Drug Metab. Pharmacokinet. 2015, 30, 127-129. [CrossRef]

79. Hiratsuka, M.; Yamashita, H.; Akai, F.; Hosono, H.; Hishinuma, E.; Hirasawa, N.; Mori, T. Genetic polymorphisms of dihydropyrimidinase in a Japanese patient with capecitabine-induced toxicity. PLoS ONE 2015, 10, e0124818. [CrossRef]

80. Fidlerova, J.; Kleiblova, P.; Bilek, M.; Kormunda, S.; Formankova, Z.; Novotny, J.; Kleibl, Z. Contribution of dihydropyrimidinase gene alterations to the development of serious toxicity in fluoropyrimidine-treated cancer patients. Cancer Chemother. Pharmacol. 2010, 65, 661-669. [CrossRef]

81. Nakajima, Y.; Meijer, J.; Zhang, C.; Wang, X.; Kondo, T.; Ito, T.; Dobritzsch, D.; Van Kuilenburg, A.B. Altered Pre-mRNA Splicing Caused by a Novel Intronic Mutation c.1443+5G>A in the Dihydropyrimidinase (DPYS) Gene. Int. J. Mol. Sci. 2016, 17, 86. [CrossRef] [PubMed] 
82. Thomas, H.R.; Ezzeldin, H.H.; Guarcello, V.; Mattison, L.K.; Fridley, B.L.; Diasio, R.B. Genetic regulation of dihydropyrimidinase and its possible implication in altered uracil catabolism. Pharm. Genom. 2007, 17, 973-987. [CrossRef] [PubMed]

83. Hishinuma, E.; Akai, F.; Narita, Y.; Maekawa, M.; Yamaguchi, H.; Mano, N.; Oda, A.; Hirasawa, N.; Hiratsuka, M. Functional characterization of 21 allelic variants of dihydropyrimidinase. Biochem. Pharmacol. 2017, 143, 118-128. [CrossRef] [PubMed]

84. Hsieh, Y.C.; Chen, M.C.; Hsu, C.C.; Chan, S.I.; Yang, Y.S.; Chen, C.J. Crystal structures of vertebrate dihydropyrimidinase and complexes from Tetraodon nigroviridis with lysine carbamylation: Metal and structural requirements for post-translational modification and function. J. Biol. Chem. 2013, 288, 30645-30658. [CrossRef]

85. Brooks, K.P.; Jones, E.A.; Kim, B.D.; Sander, E.G. Bovine liver dihydropyrimidine amidohydrolase: Purification, properties, and characterization as a zinc metalloenzyme. Arch. Biochem. Biophys. 1983, 226, 469-483. [CrossRef]

86. Lohkamp, B.; Andersen, B.; Piskur, J.; Dobritzsch, D. The crystal structures of dihydropyrimidinases reaffirm the close relationship between cyclic amidohydrolases and explain their substrate specificity. J. Biol. Chem. 2006, 281, 13762-13776. [CrossRef]

87. Tzeng, C.T.; Huang, Y.H.; Huang, C.Y. Crystal structure of dihydropyrimidinase from Pseudomonas aeruginosa PAO1: Insights into the molecular basis of formation of a dimer. Biochem. Biophys. Res. Commun. 2016, 478, 1449-1455. [CrossRef]

88. Kim, G.J.; Kim, H.S. C-terminal regions of D-hydantoinases are nonessential for catalysis, but affect the oligomeric structure. Biochem. Biophys. Res. Commun. 1998, 243, 96-100. [CrossRef]

89. Niu, L.; Zhang, X.; Shi, Y.; Yuan, J. Subunit dissociation and stability alteration of D hydantoinase deleted at the terminal amino acid residue. Biotechnol. Lett. 2007, 29, 303-308. [CrossRef]

90. Abendroth, J.; Niefind, K.; Schomburg, D. X-ray structure of a dihydropyrimidinase from Thermus sp. at 1.3 A resolution. J. Mol. Biol. 2002, 320, 143-156. [CrossRef]

91. Gojkovic, Z.; Rislund, L.; Andersen, B.; Sandrini, M.P.; Cook, P.F.; Schnackerz, K.D.; Piskur, J. Dihydropyrimidine amidohydrolases and dihydroorotases share the same origin and several enzymatic properties. Nucleic Acids Res. 2003, 31, 1683-1692. [CrossRef] [PubMed]

92. Sakamoto, T.; Sakata, S.F.; Matsuda, K.; Horikawa, Y.; Tamaki, N. Expression and properties of human liver beta-ureidopropionase. J. Nutr. Sci. Vitaminol. 2001, 47, 132-138. [CrossRef] [PubMed]

93. van Kuilenburg, A.B.; Meinsma, R.; Beke, E.; Assmann, B.; Ribes, A.; Lorente, I.; Busch, R.; Mayatepek, E.; Abeling, N.G.; van Cruchten, A.; et al. beta-Ureidopropionase deficiency: An inborn error of pyrimidine degradation associated with neurological abnormalities. Hum. Mol. Genet. 2004, 13, 2793-2801. [CrossRef] [PubMed]

94. Akiyama, T.; Shibata, T.; Yoshinaga, H.; Kuhara, T.; Nakajima, Y.; Kato, T.; Maeda, Y.; Ohse, M.; Oka, M.; Kageyama, M.; et al. A Japanese case of $\beta$-ureidopropionase deficiency with dysmorphic features. Brain Dev. 2017, 39, 58-61. [CrossRef]

95. van Kuilenburg, A.B.; Dobritzsch, D.; Meijer, J.; Krumpel, M.; Selim, L.A.; Rashed, M.S.; Assmann, B.; Meinsma, R.; Lohkamp, B.; Ito, T.; et al. B-ureidopropionase deficiency: Phenotype, genotype and protein structural consequences in 16 patients. Biochim. Biophys. Acta 2012, 1822, 1096-1108. [CrossRef] [PubMed]

96. Lee, J.H.; van Kuilenburg, A.B.; Abeling, N.G.; Vasta, V.; Hahn, S.H. A Korean Case of $\beta$-Ureidopropionase Deficiency Presenting with Intractable Seizure, Global Developmental Delay, and Microcephaly. JIMD Rep. 2015, 19, 117-121. [CrossRef]

97. Nakajima, Y.; Meijer, J.; Dobritzsch, D.; Ito, T.; Meinsma, R.; Abeling, N.G.; Roelofsen, J.; Zoetekouw, L.; Watanabe, Y.; Tashiro, K.; et al. Clinical, biochemical and molecular analysis of 13 Japanese patients with beta-ureidopropionase deficiency demonstrates high prevalence of the c.977G > A (p.R326Q) mutation [corrected]. J. Inherit. Metab. Dis. 2014, 37, 801-812. [CrossRef]

98. Yaplito-Lee, J.; Pitt, J.; Meijer, J.; Zoetekouw, L.; Meinsma, R.; van Kuilenburg, A.B. Beta-ureidopropionase deficiency presenting with congenital anomalies of the urogenital and colorectal systems. Mol. Genet. Metab. 2008, 93, 190-194. [CrossRef]

99. Fang, Y.; Cai, C.; Wang, C.; Sun, B.; Zhang, X.; Fan, W.; Hu, W.; Meng, Y.; Lin, S.; Zhang, C.; et al. Clinical and genetic analysis of 7 Chinese patients with beta-ureidopropionase deficiency. Medicine 2019, 98, e14021. [CrossRef] 
100. Shu, J.; Lv, X.; Jiang, S.; Zhang, Y.; Zhang, C.; Meng, Y.; Situ, A.; Xu, H.; Song, L. Genetic analysis of the UPB1 gene in two new Chinese families with beta-ureidopropionase deficiency and the carrier frequency of the mutation c.977G >A in Northern China. Child's Nerv. Syst. Off. J. Int. Soc. Pediatric Neurosurg. 2014, 30, 2109-2114. [CrossRef]

101. Thomas, H.R.; Ezzeldin, H.H.; Guarcello, V.; Mattison, L.K.; Fridley, B.L.; Diasio, R.B. Genetic regulation of beta-ureidopropionase and its possible implication in altered uracil catabolism. Pharm. Genom. 2008, 18, 25-35. [CrossRef] [PubMed]

102. Fidlerova, J.; Kleiblova, P.; Kormunda, S.; Novotny, J.; Kleibl, Z. Contribution of the beta-ureidopropionase (UPB1) gene alterations to the development of fluoropyrimidine-related toxicity. Pharmacol. Rep. 2012, 64, 1234-1242. [CrossRef]

103. Matsusaka, S.; Lenz, H.J. Pharmacogenomics of fluorouracil -based chemotherapy toxicity. Expert Opin. Drug Metab. Toxicol. 2015, 11, 811-821. [CrossRef] [PubMed]

104. de Bock, C.E.; Garg, M.B.; Scott, N.; Sakoff, J.A.; Scorgie, F.E.; Ackland, S.P.; Lincz, L.F. Association of thymidylate synthase enhancer region polymorphisms with thymidylate synthase activity in vivo. Pharm. J. 2011, 11, 307-314. [CrossRef]

105. Amirfallah, A.; Kocal, G.C.; Unal, O.U.; Ellidokuz, H.; Oztop, I.; Basbinar, Y. DPYD, TYMS and MTHFR Genes Polymorphism Frequencies in a Series of Turkish Colorectal Cancer Patients. J. Pers. Med. 2018, 8, 45. [CrossRef]

106. Lima, A.; Azevedo, R.; Sousa, H.; Seabra, V.; Medeiros, R. Current approaches for TYMS polymorphisms and their importance in molecular epidemiology and pharmacogenetics. Pharmacogenomics 2013, 14, 1337-1351. [CrossRef]

107. Loganayagam, A.; Arenas Hernandez, M.; Corrigan, A.; Fairbanks, L.; Lewis, C.M.; Harper, P.; Maisey, N.; Ross, P.; Sanderson, J.D.; Marinaki, A.M. Pharmacogenetic variants in the DPYD, TYMS, CDA and MTHFR genes are clinically significant predictors of fluoropyrimidine toxicity. Br. J. Cancer 2013, 108, 2505-2515. [CrossRef]

108. Amstutz, U.; Offer, S.M.; Sistonen, J.; Joerger, M.; Diasio, R.B.; Largiader, C.R. Polymorphisms in MIR27A Associated with Early-Onset Toxicity in Fluoropyrimidine-Based Chemotherapy. Clin. Cancer Res. Off. J. Am. Assoc. Cancer Res. 2015, 21, 2038-2044. [CrossRef]

109. Meulendijks, D.; Henricks, L.M.; Amstutz, U.; Froehlich, T.K.; Largiader, C.R.; Beijnen, J.H.; de Boer, A.; Deenen, M.J.; Cats, A.; Schellens, J.H. Rs895819 in MIR27A improves the predictive value of DPYD variants to identify patients at risk of severe fluoropyrimidine-associated toxicity. Int. J. Cancer 2016, 138, 2752-2761. [CrossRef] [PubMed]

110. Offer, S.M.; Butterfield, G.L.; Jerde, C.R.; Fossum, C.C.; Wegner, N.J.; Diasio, R.B. microRNAs miR-27a and miR-27b directly regulate liver dihydropyrimidine dehydrogenase expression through two conserved binding sites. Mol. Cancer Ther. 2014, 13, 742-751. [CrossRef] [PubMed] 